hep-th/9705039

DESY 97-077

May 1997

\title{
CFT FUSION RULES, DHR GAUGE GROUPS, AND CAR ALGEBRAS
}

\author{
Jens Böckenhauer \\ University of Wales Swansea, Department of Mathematics \\ Singleton Park, Swansea SA2 8PP \\ Jürgen Fuchs $x$ \\ DESY \\ Notkestraße 85, D - 22603 Hamburg
}

\begin{abstract}
.
It is demonstrated that several series of conformal field theories, while satisfying braid group statistics, can still be described in the conventional setting of the DHR theory, i.e. their superselection structure can be understood in terms of a compact DHR gauge group. Besides theories with only simple sectors, these include (the untwisted part of) $c=1$ orbifold theories and level two $\widehat{\mathfrak{s o}}(2 N)$ WZW theories. We also analyze the relation between these models and theories of complex free fermions.
\end{abstract}

$X$ Heisenberg fellow 


\section{Introduction}

In local relativistic quantum field theory, the fusion rules encode the basis independent features of the composition of superselection sectors. When the theory enjoys permutation group statistics, as is e.g. the case in four space-time dimensions, then - under standard assumptions which are motivated by physical principles such as causality (see e.g. [1]) the fusion rules can be studied with the help of the DHR theory [2, 3, 4], implying that the composition of sectors is governed by a compact group [5], the DHR gauge group. More precisely, the fusion ring of the theory is isomorphic to the representation ring of the gauge group. On the other hand, quantum field theories in space-times of low dimensionality generically possess braid group statistics. As a consequence the rôle of the gauge group is taken over by a much more complicated structure, which is commonly called a quantum symmetry, and for which no generally accepted description is available yet.

The purpose of this paper is to demonstrate that several classes of low-dimensional quantum field theories, while satisfying braid group statistics, can nevertheless be described in the conventional DHR setting. The models in question are certain rational conformal field theories. A necessary prerequisite for such a description to work is that the statistical dimensions $d_{a}$ of all sectors are integers,

$$
d_{a} \in \mathbb{Z} \text { for all } a \text {. }
$$

In the particular case that all sectors are simple in the sense that they have statistical dimension 1, the relevant braid group representation is one-dimensional and the fusion ring is nothing but the group ring of a finite abelian group. For these 'abelian' theories it is rather straightforward to interpret the relevant abelian group as a DHR gauge group.

However, as we will show in this paper, a DHR interpretation is even possible for series of rational quantum field theories which satisfy (1.1), but for which sectors with statistical dimension larger than 1 appear. More specifically, we consider円 series of models which are labelled by a natural number $N \in \mathbb{N}$, such that there are four sectors of statistical dimension 1 and $N-1$ sectors of statistical dimension 2. In conformal field theory, two different realizations of this series are known: first, the untwisted/2 sectors of 'orbifold theories' of conformal central charge $c=1$ (see e.g. [7]), and second, the untwisted sectors of WZW theories [8] that are based on the $\widehat{\mathfrak{s o}}(2 N)$ current algebra at level 2, which have $c=2 N-1$. Both of these realizations can be understood in terms of a reduction of other series of conformal field theories which have $2 N$ sectors, each of statistical dimension 1 , namely of the $c=1$ theories that describe a free boson compactified on a circle of suitable radius, and of the $c=2 N-1$ WZW theories based on $\widehat{\mathfrak{s l}}(2 N)$ at level 1 , respectively. Accordingly, we will start our discussion with an analysis of those 'abelian' theories.

\footnotetext{
${ }^{1}$ Another example has been studied in the appendix of [6].

2 The term 'untwisted' refers to a $\mathbb{Z}_{2}$-gradation of the full fusion ring of these conformal field theories. The $N-1$ untwisted sectors form a sub-fusion ring of the full fusion ring, and it is this subring we consider here. In addition there are four twisted sectors of statistical dimension $\sqrt{N}$. Clearly, with our methods we cannot study these twisted sectors, except possibly when $N$ is a square (for the latter case see the speculations at the end of the paper). Note, however, that in the DHR framework no recourse to concepts like modular invariance which do not have an immediate physical interpretation is needed. Accordingly, while the twisted sectors must be included when one wishes to construct a modular invariant two-dimensional conformal field theory, in our present study of one-dimensional chiral conformal field theories we are free to restrict to the sub-fusion ring of our interest.
} 
As it turns out, all the theories just mentioned are closely related to theories of complexp free fermions. In establishing these relations, an important rôle will be played by various types of DHR gauge groups. We would like to mention that the description in terms of fermions has several advantages with respect to a formulation via free bosons, which for these theories exists as well. For instance, one only has to deal with polynomials in the basic Fourier modes rather than with the exponentials that appear in the vertex operators of the bosonic formulation. In particular, there is a rather simple characterization of the observables, which will be described in section 6 .

Concerning the free fermion theories, it is worth while to be a bit more specific already at this introductory stage. We consider a Fock representation of the canonical anti-commutation relations (CAR) which is characterized as follows. We fix (once and for all) a positive integer $N$. On the Fock space $\mathcal{H}^{(\mathrm{CAR})} \equiv \mathcal{H}^{(\mathrm{CAR}, 2 N)}$ there operate the Fourier modes $b_{r}^{i}$ and $c_{r}^{i}(i \in\{1,2, \ldots, 2 N\}$ and $r \in \mathbb{Z}+1 / 2)$ of $2 N$ complex free fermions. These modes satisfy the relations

$$
\begin{aligned}
& \left\{b_{r}^{i}, c_{s}^{j}\right\}=\delta_{i, j} \delta_{r,-s} \mathbf{1}, \\
& \left\{b_{r}^{i}, b_{s}^{j}\right\}=0=\left\{c_{r}^{i}, c_{s}^{j}\right\},
\end{aligned}
$$

and there is a ${ }^{*}$-operation (an involutive automorphism), which acts on the modes as

$$
\left(b_{r}^{i}\right)^{*}=c_{-r}^{i} .
$$

The Fock space $\mathcal{H}^{\text {(CAR) }}$ itself is defined by the properties that it contains a unique (up to a phase) vacuum vector $|\Omega\rangle \in \mathcal{H}^{(\mathrm{CAR})}$ on which the modes $b_{r}^{i}$ and $c_{r}^{i}$ with positive index $r$ act as annihilation operators, i.e. for all $i=1,2, \ldots, 2 N$ and all $r \in \mathbb{N}_{0}+1 / 2$ we have $b_{r}^{i}|\Omega\rangle=c_{r}^{i}|\Omega\rangle=0$, while the modes with negative index $r$ act as creation operators such that their successive action on the vacuum provides a dense subspace.

Given the fermion Fourier modes $b_{r}^{i}$ and $c_{r}^{i}$, we can define local and global CAR algebras as follows. For $f \in L^{2}\left(S^{1} ; \mathbb{C}^{2 N}\right)$ we set

$$
b(f):=\sum_{i=1}^{2 N} \sum_{r \in \mathbb{Z}+1 / 2} f_{-r}^{i} b_{r}^{i} \quad \text { and } \quad c(f):=\sum_{i=1}^{2 N} \sum_{r \in \mathbb{Z}+1 / 2} f_{-r}^{i} c_{r}^{i}
$$

where $f^{i}(z)=\sum_{r \in \mathbb{Z}+1 / 2} f_{-r}^{i} z^{r}$ is the Fourier decomposition of the $i$ th component of the function $f$ (the circle $S^{1}$ is considered as the unit circle in the complex plane, so that $z=\mathrm{e}^{\mathrm{i} \phi} \in S^{1}$ with $\left.-\pi<\phi \leq \pi\right)$. For open intervals $I \subset S^{1}$ we define the local CAR algebras $\operatorname{CAR}(I)$ to be the von Neumann algebras generated by elements $b(f)$ and $c(g)$ with $f, g$ having support in $I$,

$$
\operatorname{CAR}(I):=\left\{b(f), c(g) \mid f, g \in L^{2}\left(I ; \mathbb{C}^{2 N}\right)\right\}^{\prime \prime}
$$

(The prime stands for the commutant in the algebra of bounded operators on $\mathcal{H}^{(\mathrm{CAR})}$. Note that the bicommutant of a set of operators then actually coincides with the von Neumann algebra they generate.) By construction, for any pair of open intervals $I_{1}, I_{2}$ with $I_{1} \subset I_{2}$

\footnotetext{
3 There is one other known series of rational fusion rings satisfying (1.1), namely those describing the untwisted sectors of the level two $\widehat{\mathfrak{s o}}(2 N+1)$ WZW theory. Using the results of [9], these could be analyzed along similar lines, but involving an odd number of real free fermions. However, some of the arguments turn out to become more involved, and we refrain from delving into these complications here.
} 
we have an inclusion $\operatorname{CAR}\left(I_{1}\right) \subset \mathrm{CAR}\left(I_{2}\right)$, which is inherited from the natural embeddings of the $L^{2}$ spaces. The global CAR algebra is defined as the norm closure

$$
\text { CAR }:=\overline{\bigcup_{I \in \mathcal{J}^{*}} \operatorname{CAR}(I)}
$$

of the union of local algebras, where $\mathcal{J}^{*}$ denotes the set of those open intervals $I \subset S^{1}$ whose closure does not contain the point (say) $z=-1$.

This paper is organized as follows. In section 2 we summarize some features of the DHR theory and study the interplay between the sector decompositions that arise from embeddings of gauge groups. In section 3 we present the sector decompositions of the $c=1$ 'circle' conformal field theories with $2 N$ sectors and of the level one $\widehat{\mathfrak{s l}}(2 N)$ WZW theories and interpret them in terms of DHR gauge groups $\mathbb{Z}_{2 N}$. The relation between these theories and the CAR algebras defined by (1.2) is demonstrated in section 1 . When the $\mathbb{Z}_{2 N}$ gauge symmetry of these theories is extended by a suitable further automorphism, one arrives at a description of the $c=1$ orbifold and the level two $\widehat{\mathfrak{s o}}(2 N)$ WZW theories with $N+7$ sectors; these theories and their connection with the CAR algebra is analyzed in section 5. Finally, in section 6 we present the Fourier modes of the observables, expressed in terms of the Fourier modes of the free fermions.

\section{DHR sectors and embeddings of gauge groups}

Let us briefly recall some facts about the DHR theory [2, 3, [4] of superselection sectors. We are dealing with chiral conformal field theories, so that the relevant space-time is $S^{1}$, the unit circle. To apply the DHR theory to this situation, one associates to each interval $I \subset S^{1}$ a local field algebra $\mathfrak{F}(I)$; this is a von Neumann algebra which acts on some Hilbert

space $\mathcal{H}$ in such a way that $I_{1} \subset I_{2}$ implies $\mathfrak{F}\left(I_{1}\right) \subset \mathfrak{F}\left(I_{2}\right)$. The global (or quasilocal) field algebra, which is defined as the norm closure

$$
\mathfrak{F}=\overline{\bigcup_{I \in \mathcal{J}^{*}} \mathfrak{F}(I)}
$$

of the union of all local field algebras, acts irreducibly on $\mathcal{H}$. Here as in (1.6) $\mathcal{J}^{*}$ denotes the set of open intervals in $S^{1}$ whose closure does not contain $-1 \in S^{1}$. The Hilbert space $\mathcal{H}$ carries a strongly continuous representation $R$ of the space-time symmetry group $\mathrm{SU}(1,1)$ such that the generator $L_{0}$ of rotations is positive and the eigenvalue zero belongs to a unique (up to a phase) vacuum vector $|\Omega\rangle \in \mathcal{H}$ (see e.g. [10,11]). The field algebras transform covariantly with respect to $R$. Furthermore, $\mathcal{H}$ also carries a strongly continuous representation $U$ of a compact group $G$, called the DHR gauge group, which commutes with $R$ and leaves the vacuum vector invariant, and which transforms each local field algebra into itself. Local observable algebras are the fixed point algebras of the field algebras with respect to the gauge group $G$,

$$
\mathfrak{A}(I)=\mathfrak{F}(I)^{G}:=\mathfrak{F}(I) \cap U(G)^{\prime},
$$

and the global observable algebra is

$$
\mathfrak{A}=\overline{\bigcup_{I \in \mathcal{J}^{*}} \mathfrak{A}(I)},
$$


so that $\mathfrak{A}=\mathfrak{F}^{G}$. Note that while all local algebras are von Neumann algebras, the global algebras are only $C^{*}$-algebras. Fields are relatively local to the observables, and this implies in particular locality of the observables.

Under these (and a few further standard) assumptions, the DHR theory tells us that the Hilbert space $\mathcal{H}$ decomposes as

$$
\mathcal{H}=\bigoplus_{\alpha \in \hat{G}} \mathcal{H}_{\alpha} \otimes \mathbb{C}^{d_{\alpha}}
$$

with respect to the action of $\mathfrak{A}$. Here $\mathcal{H}_{\alpha}$ are pairwise inequivalent irreducible $\mathfrak{A}$-modules, called the superselection sectors, $\hat{G}$ denotes the group dual of $G$ (i.e. the set of irreducible characters of $G$, which constitutes a basis of the representation ring of $G$ ), and $d_{\alpha}$ is the dimension of the irreducible $G$-representation $\pi_{\alpha}$ with character $\alpha \in \hat{G}$. The gauge group $G$ acts on the multiplicity space $\mathbb{C}^{d_{\alpha}}$ by the representation $\pi_{\alpha}$, i.e.

$$
U(g)=\bigoplus_{\alpha \in \hat{G}} \mathbf{1}_{\mathcal{H}_{\alpha}} \otimes \pi_{\alpha}(g) \quad \text { for all } g \in G .
$$

Next we investigate what happens when we are given two different DHR gauge groups $G$ and $H$ which act on one and the same field algebra $\mathfrak{F}$. Then there are two decompositions

$$
\bigoplus_{\alpha \in \hat{G}} \mathcal{H}_{\alpha} \otimes \mathbb{C}^{d_{\alpha}}=\mathcal{H}=\bigoplus_{a \in \hat{H}} \mathcal{H}_{a} \otimes \mathbb{C}^{d_{a}}
$$

of the Hilbert space $\mathcal{H}$ with respect to the fixed point algebras $\mathfrak{F}^{G}$ and $\mathfrak{F}^{H}$, respectively. Now consider the situation that $H \subset G$ and that the action of $H$ is defined by the restriction of $U$ from $G$ to $H$. It is not hard to see that the decompositions (2.6) are then related as

$$
\mathcal{H}_{a}=\bigoplus_{\alpha \in \hat{G}} \mathcal{H}_{\alpha} \otimes \mathbb{C}^{n_{\alpha}^{a}}
$$

where the branching coefficients $n_{\alpha}^{a}$ are defined through the restriction

$$
\operatorname{res}_{H}^{G}\left(\pi_{\alpha}\right)=\bigoplus_{a \in \hat{H}} n_{\alpha}^{a} \pi_{a}
$$

of irreducible $G$-representations $\pi_{\alpha}$ to $H$-representations, or equivalently, through

$$
\operatorname{ind}_{H}^{G}\left(\pi_{a}\right)=\bigoplus_{\alpha \in \hat{G}} n_{\alpha}^{a} \pi_{\alpha}
$$

by Frobenius reciprocity. In other words, the superselection sectors $\mathcal{H}_{a}$, labelled by $a \in \hat{H}$, are related to the sectors $\mathcal{H}_{\alpha}, \alpha \in \hat{G}$, according to the induction from $H$ to $G$.

We are particularly interested in the specific case where $H$ is embedded diagonally into $G=H \times H$. Then $\hat{G}=\hat{H} \times \hat{H}$, so that $\alpha \in \hat{G}$ can be considered as a pair $\left(a_{1}, a_{2}\right)$ with $a_{1}, a_{2} \in \hat{H}$. It follows that

$$
n_{\alpha}^{a} \equiv n_{\left(a_{1}, a_{2}\right)}^{a}=N_{a_{1} a_{2}}^{a},
$$

where $N_{a b}{ }^{c}$ are the fusion coefficients of $H$, defined by the tensor product decomposition

$$
\pi_{a} \otimes \pi_{b} \cong \bigoplus_{c \in \hat{H}} N_{a b}^{c} \pi_{c}
$$


of irreducible $H$-representations. This observation applies in particular to the situation where the field algebra $\mathfrak{F}$ has the structure of a tensor product $\mathfrak{F}=\mathfrak{F}_{(1)} \otimes \mathfrak{F}_{(2)}$ of field algebras which possess isomorphic DHR gauge groups, i.e. for which the associated observable algebras are

$$
\mathfrak{A}_{(1)}=\left(\mathfrak{F}_{(1)}\right)^{H_{(1)}}, \quad \mathfrak{A}_{(2)}=\left(\mathfrak{F}_{(2)}\right)^{H_{(2)}} \quad \text { with } \quad H_{(1)} \cong H_{(2)} \cong: H .
$$

In this case the field algebra $\mathfrak{F}$ acts in a canonical manner on the tensor product $\mathcal{H}=\mathcal{H}^{(1)} \otimes$ $\mathcal{H}^{(2)}$ of Hilbert spaces $\mathcal{H}^{(1)}$ and $\mathcal{H}^{(2)}$, which under the action of the observable algebras $\mathfrak{A}_{(1)}$ and $\mathfrak{A}_{(2)}$ decompose into sectors as

$$
\mathcal{H}^{(1)}=\bigoplus_{a \in \hat{H}} \mathcal{H}_{a}^{(1)} \otimes \mathbb{C}^{d_{a}} \quad \text { and } \quad \mathcal{H}^{(2)}=\bigoplus_{a \in \hat{H}} \mathcal{H}_{a}^{(2)} \otimes \mathbb{C}^{d_{a}}
$$

respectively. It follows from the result above that under the action of the diagonal subgroup of $H_{(1)} \times H_{(2)}, \mathcal{H}$ decomposes as

$$
\mathcal{H}=\bigoplus_{a \in \hat{H}} \mathcal{H}_{a} \otimes \mathbb{C}^{d_{a}} \quad \text { with } \quad \mathcal{H}_{a}=\bigoplus_{b, c \in \hat{H}} \mathcal{H}_{b}^{(1)} \otimes \mathcal{H}_{c}^{(2)} \otimes \mathbb{C}^{N_{b c}{ }^{a}} .
$$

Below we will encounter the specific case of cyclic gauge group $H \cong \mathbb{Z}_{2 N} \equiv \mathbb{Z} / 2 N \mathbb{Z}$. Then also $\hat{H} \cong \mathbb{Z}_{2 N}$; thus the labels $a \in \hat{H}$ can (and will) be considered as integers defined modulo $2 N$, i.e. $\hat{H}=\{0,1, \ldots, 2 N-1\}$, and the fusion coefficients read $N_{a b}{ }^{c}=\delta_{a+b, c}$ for $a, b, c \in \hat{H}$. Therefore the decomposition (2.14) reads $\mathcal{H}=\bigoplus_{a \in \mathbb{Z}_{2 N}} \mathcal{H}_{a}$ with

$$
\mathcal{H}_{a}=\bigoplus_{b \in \mathbb{Z}_{2 N}} \mathcal{H}_{b}^{(1)} \otimes \mathcal{H}_{a-b}^{(2)}
$$

in particular, the vacuum sector $\mathcal{H}_{0}$ splits as

$$
\mathcal{H}_{0}=\bigoplus_{a \in \mathbb{Z}_{2 N}} \mathcal{H}_{a}^{(1)} \otimes \mathcal{H}_{2 N-a}^{(2)}
$$

\section{$3 \quad c=1$ and WZW theories with $\mathbb{Z}_{2 N}$ fusion rules}

For intervals $I \subset S^{1}$ we denote by $\operatorname{Bos}_{N}(I)$ the local observable algebras of the $c=1$ conformal field theory with $2 N$ sectors that corresponds to a (chiral) free boson compactified on a circle of appropriate radius. According to the results of [12], the algebras $\operatorname{Bos}_{N}(I)$ are the von Neumann algebras that are generated by local bounded functions of a $\widehat{\mathfrak{u}}(1)$ current and of a conjugate pair of Virasoro-primary fields of conformal dimension $\Delta=N$. Similarly, we denote by $\mathrm{SL}_{2 N}(I)$ the local observable algebras of the WZW theory based on the $\widehat{\mathfrak{s l}}(2 N)$ current algebra at level 1 . The corresponding global $C^{*}$-algebras will be denoted by $\operatorname{Bos}_{N}$ and $\mathrm{SL}_{2 N}$, respectively, and the associated field algebras by $\mathfrak{F}_{(\text {Bos })} \equiv \mathfrak{F}_{\text {(Bos;2N) }}$ and $\mathfrak{F}_{(\mathrm{SL})} \equiv \mathfrak{F}_{(\mathrm{SL} ; 2 N)}$, respectively. Unfortunately, while explicit expressions for localized fields are available in the $c=1$ case $[12]$, to the best of our knowledge they are not known for the WZW theories. However, both for the $c=1$ and the WZW theories, 'point-like localized' unbounded field operators can be obtained by the vertex operator construction. For the $c=1$ theory the vertex operators are given by

$$
\varphi_{a}(z) \sim: \mathrm{e}^{\mathrm{i} a X(z) / \sqrt{2 N}}:
$$


for $z \in S^{1}$, while for the WZW case they read

$$
\phi_{a}(z) \sim: \mathrm{e}^{\mathrm{i}\left(\Lambda_{(a)}, Y(z)\right)}: .
$$

Here $a \in \mathbb{Z}_{2 N}, X$ is a free boson and $Y \equiv\left(Y^{i}\right)$ a collection of $2 N-1$ free bosons, and the colons stand for a suitable normal ordering prescription; moreover, $\Lambda_{(a)}$ denote the fundamental weights of the finite-dimensional Lie algebra $\mathfrak{s l}(2 N)$ (for $a \neq 0$, together with $\left.\Lambda_{(0)}:=0\right)$, and $(\cdot, \cdot)$ is the inner product on the weight space of $\mathfrak{s l}(2 N)$.

Both of these two types of quantum field theories possess $2 N$ sectors, each of which has statistical dimension one, and in either case the fusion ring is the group ring of the finite cyclic group $\mathbb{Z}_{2 N}$. In this situation it is quite natural to expect that the composition of sectors can be understood by promoting this $\mathbb{Z}_{2 N}$ group to a DHR gauge group; the following considerations demonstrate that this is indeed the case. (Similar arguments will work for any other theory whose fusion ring coincides with the group ring of a finite abelian group.)

The sectors of the $c=1$ and of the WZW theories can be obtained by applying the Fourier modes of the observables of these theories to suitable highest weight vectors; in both cases it is in fact sufficient to employ only the generators of the relevant current algebras (which will be described in detail in section 6). It follows that the sectors of these theories are isomorphic, respectively, to direct sums of the irreducible highest weight modules $L_{b}^{(\mathfrak{u}(1))}$ of the $\widehat{\mathfrak{u}}(1)$ current algebra with charge $b \bmod 2 N$, and to the irreducible highest weight modules $L_{\Lambda}^{(\mathrm{SL})} \equiv L_{\Lambda}^{(\mathrm{SL} ; 2 N)}$ of the WZW theory with certain specific highest $\mathfrak{s l}(2 N)$-weights $\Lambda$. More specifically, one finds that the sectors $L_{b}^{(\mathrm{Bos})} \equiv L_{b}^{(\mathrm{Bos} ; 2 N)}$ of the $c=1$ theory are the direct sums

$$
L_{b}^{(\mathrm{Bos})}=\bigoplus_{n \in \mathbb{Z}} L_{b+2 n N}^{(\mathfrak{u}(1))}
$$

while the sectors $L_{\Lambda_{(a)}}^{(\mathrm{SL})}$ of the level one $\mathfrak{s l}(2 N)$ theory are modules $L_{\Lambda}^{(\mathrm{SL})}$ whose highest weight $\Lambda$ is either $\Lambda_{(0)}=0$ (for the vacuum sector) or a fundamental weight $\Lambda_{(a)}(a \in$ $\{1,2, \ldots, 2 N-1\})$ of $\mathfrak{s l}(2 N)$. Thus the sector decompositions of these theories read

$$
\mathcal{H}^{(\mathrm{Bos} ; 2 N)}=\bigoplus_{a \in \mathbb{Z}_{2 N}} L_{a}^{(\mathrm{Bos})} \equiv \bigoplus_{a \in \mathbb{Z}_{2 N}} \bigoplus_{n \in \mathbb{Z}} L_{a+2 n N}^{(\mathfrak{u}(1))}=\bigoplus_{m \in \mathbb{Z}} L_{m}^{(\mathfrak{u}(1))}
$$

and

$$
\mathcal{H}^{(\mathrm{SL} ; 2 N)}=\bigoplus_{a \in \mathbb{Z}_{2 N}} L_{\Lambda_{(a)}^{(\mathrm{SL})}}
$$

respectively.

From these decompositions we learn in particular that the spaces $\mathcal{H}^{(\mathrm{Bos} ; 2 N)}$ and $\mathcal{H}^{(\mathrm{SL} ; 2 N)}$ naturally carry representations of $\mathbb{Z}_{2 N}$. Since the group $\mathbb{Z}_{2 N}$ does not possess a distinguished generator, there is a priori some arbitrariness in the precise definition of these

${ }^{4}$ To be precise, this actually yields only dense subspaces of the sectors (the same remark applies to the sectors of the other theories treated below). However, this will not play any rôle in our discussion, and accordingly we simplify notation by using the same symbols for the sectors and for their dense subspaces.

5 The individual summands are related to each other by the action of the additional observables of conformal weight $N$. For the present statements we do not, however, need any information about the form of this action. 
representations, though. As it turns out, a convenient prescription for the representations $U^{(\mathrm{Bos})} \equiv U^{(\mathrm{Bos} ; 2 N)}$ of $\mathbb{Z}_{2 N}$ on $\mathcal{H}^{(\mathrm{Bos} ; 2 N)}$ and $U^{(\mathrm{SL})} \equiv U^{(\mathrm{SL} ; 2 N)}$ of $\mathbb{Z}_{2 N}$ on $\mathcal{H}^{(\mathrm{SL} ; 2 N)}$ is provided by

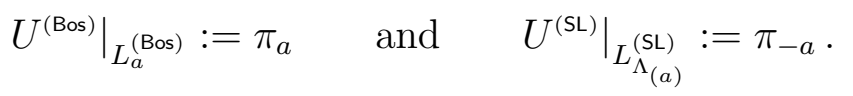

Here for each $a \in \mathbb{Z}_{2 N}, \pi_{a}$ denotes the $\mathbb{Z}_{2 N}$-representation that acts as

$$
\pi_{a}(b)=\mathrm{e}^{\mathrm{i} \pi a b / N} \quad \text { for all } b \in \mathbb{Z}_{2 N} .
$$

\section{CAR algebras from conformal field theories with $\mathbb{Z}_{2 N}$ fusion rules}

The discussion of the previous section shows in particular that we are dealing with a situation of the type described in equation (2.12) above, with

$$
\operatorname{Bos}_{N} \equiv \mathfrak{A}_{(\mathrm{Bos} ; 2 N)}=\left(\mathfrak{F}_{(\mathrm{Bos} ; 2 N)}\right)^{\mathbb{Z}_{2 N}}, \quad \mathrm{SL}_{2 N} \equiv \mathfrak{A}_{(\mathrm{SL} ; 2 N)}=\left(\mathfrak{F}_{(\mathrm{SL} ; 2 N)}\right)^{\mathbb{Z}_{2 N}}
$$

It is then natural to ask what the fixed point algebra of $\mathfrak{F} \equiv \mathfrak{F}_{(\text {Bos;2N) }} \otimes \mathfrak{F}_{(\mathrm{SL} ; 2 N)}$ with respect to the diagonal subgroup $\mathbb{Z}_{2 N}^{\text {(diag) }} \subset \mathbb{Z}_{2 N} \times \mathbb{Z}_{2 N}$ looks like. As usual, $\mathfrak{F}$ acts irreducibly on a Hilbert space $\mathcal{H}$, and under the action of $\mathfrak{F}^{\mathbb{Z}_{2 N} \text { (diag) }}$ we have a decomposition $\mathcal{H}=$ $\bigoplus_{a \in \mathbb{Z}_{2 N}} \mathcal{H}_{a}$. As it turns out, $\mathfrak{F}^{\mathbb{Z}_{2 N} \text { (diag) }}$ is nothing but the CAR algebra (1.2) for $2 N$ complex fermions, i.e.

$$
\left(\mathfrak{F}_{(\mathrm{Bos} ; 2 N)} \otimes \mathfrak{F}_{(\mathrm{SL} ; 2 N)}\right)^{\mathbb{Z}_{2 N}(\mathrm{diag})} \cong \mathrm{CAR}
$$

where it is understood that the action of $\left(\mathfrak{F}_{(\mathrm{Bos})} \otimes \mathfrak{F}_{(\mathrm{SL})}\right)^{\mathbb{Z}_{2 N}(\mathrm{diag})}$ is restricted to the vacuum sector $\mathcal{H}_{0}$ with respect to the diagonal $\mathbb{Z}_{2 N}$ subgroup. Note that (4.2) implies in particular that

$$
\begin{aligned}
\mathrm{CAR}^{\mathbb{Z}_{2 N}} \cong\left(\mathfrak{F}_{(\mathrm{Bos} ; 2 N)} \otimes \mathfrak{F}_{(\mathrm{SL} ; 2 N)}\right)^{\mathbb{Z}_{2 N} \times \mathbb{Z}_{2 N}} \\
=\left(\mathfrak{F}_{(\mathrm{Bos} ; 2 N)}\right)^{\mathbb{Z}_{2 N}} \otimes\left(\mathfrak{F}_{(\mathrm{SL} ; 2 N)}\right) \mathbb{Z}_{2 N}=\operatorname{Bos}_{N} \otimes \mathrm{SL}_{2 N} .
\end{aligned}
$$

We will study this relationship in section 6 in terms of the Fourier modes of the observables. Here we verify (4.2) in terms of the superselection sectors, i.e. show that the vacuum sector $\mathcal{H}_{0}$ on which $\left(\mathfrak{F}_{(\text {Bos })} \otimes \mathfrak{F}_{(\mathrm{SL})}\right)^{\mathbb{Z}_{2 N}(\mathrm{diag})}$ is acting coincides with $\mathcal{H}^{(\mathrm{CAR})}$. Recall from the introduction that we can construct the sectors $\mathcal{H}_{a}^{(\mathrm{CAR})}$ of the fermion theory by applying the Fourier modes of the fermions to the vacuum. Now the results on the Hilbert spaces of the $c=1$ and WZW theories that we listed in the previous section imply in particular that (a dense subspace of) the Hilbert space of the tensor product theory is

$$
\mathcal{H}=\mathcal{H}^{(\mathrm{Bos} ; 2 N)} \otimes \mathcal{H}^{(\mathrm{SL} ; 2 N)} \cong \bigoplus_{a, b \in \mathbb{Z}_{2 N}}\left[L_{a}^{(\mathrm{Bos})} \otimes L_{\left.\Lambda_{(b)}^{(\mathrm{SL})}\right]}\right]=\bigoplus_{a, b \in \mathbb{Z}_{2 N}}\left[\left(\bigoplus_{n \in \mathbb{Z}} L_{a+2 n N}^{(\mathrm{u}(1))}\right) \otimes L_{\Lambda_{(b)}}^{(\mathrm{SL})}\right]
$$

Furthermore, it is known [13] that the space $\mathcal{H}^{(\mathrm{CAR})}=\bigoplus_{a \in \mathbb{Z}_{2 N}} \mathcal{H}_{a}^{(\mathrm{CAR})}$ decomposes as

$$
\mathcal{H}^{(\mathrm{CAR})} \cong \bigoplus_{b \in \mathbb{Z}_{2 N}}\left[\left(\bigoplus_{n \in \mathbb{Z}} L_{b+2 n N}^{(\mathfrak{u}(1))}\right) \otimes L_{\Lambda_{(b)}}^{(\mathrm{SL})}\right]
$$

\footnotetext{
${ }^{6}$ We continue to use the additive notation for integers modulo $2 N$.

${ }^{7}$ Here and in the following, equalities and isomorphisms between algebras are meant to apply both to global and to local algebras.
} 
into a direct sum of tensor products of the current algebra modules that appear in the former decompositions.

We would like to compare the results (4.4) and (4.5) from the perspective of the DHR situation that we studied in section 2. To this end we employ the $\mathbb{Z}_{2 N}$-representations $U^{\text {(Bos) }}$ and $U^{(\mathrm{SL})}(3.6)$. On $\mathcal{H}$, these representations induce an action of $\mathbb{Z}_{2 N} \times \mathbb{Z}_{2 N}$ according to

$$
\mathbb{Z}_{2 N} \times \mathbb{Z}_{2 N} \ni(a, b) \mapsto U^{(\mathrm{Bos})}(a) \otimes U^{(\mathrm{SL})}(b) .
$$

We can then restrict this representation to the diagonal subgroup of $\mathbb{Z}_{2 N} \times \mathbb{Z}_{2 N}$ and decompose $\mathcal{H}$ into its sectors $\mathcal{H}_{a}$ with respect to this diagonal subgroup:

$$
\mathcal{H}=\bigoplus_{a \in \mathbb{Z}_{2 N}} \mathcal{H}_{a} \quad \text { with } \quad \mathcal{H}_{a}:=\bigoplus_{b \in \mathbb{Z}_{2 N}}\left[\left(\bigoplus_{n \in \mathbb{Z}} L_{a+b+2 n N}^{(\mathfrak{u}(1))}\right) \otimes L_{\Lambda_{(b)}}^{(\mathrm{SL})}\right]
$$

Comparison of (4.4) with (4.5) now tells us that

$$
\mathcal{H}^{(\mathrm{CAR})} \cong \bigoplus_{a \in \mathbb{Z}_{2 N}} L_{a}^{(\mathrm{Bos})} \otimes L_{\Lambda_{(a)}^{(\mathrm{SL})}}=\mathcal{H}_{0}
$$

This finally confirms the validity of the isomorphism (4.2).

Let us also mention that when we consider the action of the full group $\mathbb{Z}_{2 N} \times \mathbb{Z}_{2 N}$ instead of its diagonal subgroup, then we must have an additional $\mathbb{Z}_{2 N}$ action in the fermionic Fock space $\mathcal{H}^{(\mathrm{CAR})}$ of the $2 N$ complex fermions. To fit with the previous results, for each $a \in \mathbb{Z}_{2 N}$ such an additional gauge transformation must act on the fermion modes $b_{r}^{i}$ and $c_{r}^{i}$ as the Bogoliubov transformation

$$
\zeta_{a}: \quad b_{r}^{i} \mapsto \mathrm{e}^{\mathrm{i} \pi a / N} b_{r}^{i}, \quad c_{r}^{i} \mapsto \mathrm{e}^{-\mathrm{i} \pi a / N} c_{r}^{i} .
$$

It is illustrative to formulate the results above also in terms of the (Virasoro-specialized) characters

$$
\chi_{V}(q):=\operatorname{tr}_{V} q^{L_{0}}
$$

of the various vector spaces $V$ that appeared in the decompositions. We first note that (4.9) can be regarded as the restriction of an action of the gauge group $\mathrm{U}(1)$, for which $a \in \mathbb{Z}_{2 N}$ is just to be replaced by an arbitrary real parameter. The characters of the sectors of the algebra $\mathrm{CAR}^{\mathbb{Z}_{2 N} \text { (diag) }}$ can therefore be calculated by first considering the decomposition of $\mathcal{H}^{(\mathrm{CAR})}$ into sectors $\mathcal{H}_{m}^{[\mathrm{U}(1)]}, m \in \mathbb{Z}$, with respect to a gauge group $G=\mathrm{U}(1)$ and then the restriction of $G$ to its subgroup $H=\mathbb{Z}_{2 N}$. In this situation we can again apply the relation (2.7), which tells us that

$$
\mathcal{H}_{a}=\bigoplus_{n \in \mathbb{Z}} \mathcal{H}_{a+2 n N}^{[\mathrm{U}(1)]}
$$

for each $a \in \mathbb{Z}_{2 N}$.

Now the characters for the spaces $\mathcal{H}_{m}^{[\mathrm{U}(1)]}$ read (see e.g. [9])

$$
\chi_{m}^{[\mathrm{U}(1)]}(q)=\Theta_{m}(q)(\varphi(q))^{-2 N},
$$

while the characters of the sectors $\mathcal{H}_{a}^{(\mathrm{Bos})}$ of $\mathfrak{F}_{(\mathrm{Bos})}$ and $\mathcal{H}_{a}^{(\mathrm{SL})}$ of $\mathfrak{F}_{(\mathrm{SL})}$ are given by

$$
\chi_{a}^{(\mathrm{Bos} ; 2 N)}(q)=(\varphi(q))^{-1} \psi_{a}(q)
$$


and by

$$
\chi_{a}^{(\mathrm{SL} ; 2 N)}(q)=q^{-a^{2} / 2 N}(\varphi(q))^{1-2 N} \Theta_{a}(q),
$$

respectively. Here

$$
\varphi(q):=\prod_{n=1}^{\infty}\left(1-q^{n}\right)
$$

is Euler's product function, while

$$
\Theta_{a}(q):=\sum_{\substack{m_{1}, m_{2}, \ldots, m_{2 N} \in \mathbb{Z} \\ m_{1}+m_{2}+\ldots+m_{2 N}=a}} q^{\left(m_{1}^{2}+m_{2}^{2}+\ldots+m_{2 N}^{2}\right) / 2}
$$

and

$$
\psi_{a}(q):=\sum_{m \in \mathbb{Z}} q^{(a+2 m N)^{2} / 4 N}
$$

for $a \in \mathbb{Z}_{2 N}$. It is then easy to verify (compare e.g. formula (9.17) of [9]) that indeed

$$
\chi_{a}(q)=\sum_{n \in \mathbb{Z}} \chi_{a+2 n N}^{[\mathrm{U}(1)]}(q)=\chi_{a}^{(\mathrm{Bos} ; 2 N)}(q) \cdot \chi_{a}^{(\mathrm{SL} ; 2 N)}(q)
$$

for all $a \in \mathbb{Z}_{2 N}$, in agreement with our result that the sectors of the $\mathbb{Z}_{2 N}$ fermion algebra are precisely the tensor products of the irreducible modules for $\operatorname{Bos}_{N}$ and $\mathrm{SL}_{2 N}$.

As another consistency check of the relation (4.2) we verify that the fields in the algebra $\left(\mathfrak{F}_{(\text {Bos } ; 2 N)} \otimes \mathfrak{F}_{(\mathrm{SL} ; 2 N)}\right)^{\mathbb{Z}_{2 N}(\mathrm{diag})}$ possess the right braiding properties. Since the form of the braid relations does not depend on the precise choice of localization of the fields, we can consider the 'point-like localized' unbounded field operators (3.1) and (3.2). These vertex operators carry an abelian ('anyonic') representation of the braid group, and the phases appearing in this representation can be determined from the conformal weights of the vertex operators. More specifically, for the $c=1$ theory we have $\Delta\left(\varphi_{a}\right)=a^{2} / 4 N$, and accordingly [12] $\varphi_{a}(z) \varphi_{b}(w)=\mathrm{e}^{\mathrm{i} \pi \epsilon a b / 2 N} \varphi_{b}(w) \varphi_{a}(z)$ (with the sign $\epsilon \in\{ \pm 1\}$ in the exponent depending on whether $w$ is to the 'left' or to the 'right' of $z$ on the punctured circle), while for the WZW theory we have $\Delta\left(\phi_{a}\right)=a(2 N-a) / 4 N$ so that $\phi_{a}(z) \phi_{b}(w)=$ $\pm \mathrm{e}^{-\mathrm{i} \pi \epsilon a b / 2 N} \phi_{b}(w) \phi_{a}(z)$. Those fields in the tensor product theory which are invariant under the diagonal $\mathbb{Z}_{2 N}$ gauge group therefore all have conformal weight $a / 2$ for some $a \in \mathbb{Z}_{2 N}$ and satisfy fermionic or bosonic braiding relations, as is required for (4.2) to hold.

\section{Theories with gauge group $Q_{N}$}

It is known that the sectors of the $c=1$ circle theory with observable algebra $\operatorname{Bos}_{N}$ combine, respectively decompose, into the untwisted sectors of the $c=1$ orbifold theory that has $N+7$ sectors [7]. Moreover, inspection of the results of [7] also shows that these untwisted orbifold sectors generate a fusion ring which is isomorphic to the representation ring of the generalized quaternion group $\mathrm{Q}_{N}$ (see appendix A for some basic information about these non-abelian finite groups). Similarly, the $\mathrm{SL}_{2 N}$ sectors give rise to the untwisted sectors of the level two $\widehat{\mathfrak{s o}}(2 N)$ WZW theory, which can be seen to generate a $\mathrm{Q}_{N}$ fusion ring as well. These observations lead us to expect that, as far as the untwisted sectors are concerned, the superselection structure of the $c=1$ orbifold and level two $\widehat{\mathfrak{s o}}(2 N)$ theories can be understood in terms of a DHR gauge group $\mathrm{Q}_{N}$. In this section we show 
that indeed one obtains the observable algebras of these theories when one extends the $\mathbb{Z}_{2 N}$ gauge groups that appeared in the previous setting to $\mathrm{Q}_{N}$. Precisely speaking, we claim that we have an action of $Q_{N}$ such that

$$
\left(\mathfrak{F}_{(\mathrm{Bos} ; 2 N)}\right)^{\mathrm{Q}_{N}}=\mathrm{Orb}_{N} \quad \text { and } \quad\left(\mathfrak{F}_{(\mathrm{SL} ; 2 N)}\right)^{\mathrm{Q}_{N}}=\mathrm{SO}_{2 N},
$$

where $\mathrm{Orb}_{N}$ and $\mathrm{SO}_{2 N}$ stand for the observable algebras of the $c=1$ orbifold theory with $N+7$ sectors and of the level two $\widehat{\mathfrak{s o}}(2 N)$ WZW theory, respectively.

To prove this claim and to study its consequences, it is convenient to express the action of the gauge groups in terms of automorphisms of the relevant field algebras. For each $a \in \mathbb{Z}_{2 N}$ the representations $U^{(1)} \equiv U^{(\mathrm{Bos} ; 2 N)}$ and $U^{(2)} \equiv U^{(\mathrm{SL} ; 2 N)}$ that were introduced in (3.6) define automorphisms $\operatorname{Ad}_{U^{(j)}(a)}$ of the field algebras $\mathfrak{F}_{(j)}$ for $j=1,2$, respectively. We denote by $x$ the generator of the abstract group $\mathbb{Z}_{2 N}\left(x^{2 N}=1\right)$, and define

$$
\xi_{(j)}:=\operatorname{Ad}_{U^{(j)}(x)} \in \operatorname{Aut}\left(\mathfrak{F}_{(j)}\right)
$$

for $j \in\{1,2\}$. We wish to consider the situation that the $\mathbb{Z}_{2 N}$ gauge groups of both the $c=1$ and the WZW theory get extended to the generalized quaternion groups $\mathrm{Q}_{N}$, by including another generator $y$ in such a way that the relations (A.1) of $\mathrm{Q}_{N}$ are satisfied. This means that for $j=1,2$ we have besides $\xi_{(j)}$ automorphisms $\theta_{(j)}=\operatorname{Ad}_{U^{(j)}(y)} \in \operatorname{Aut}\left(\mathfrak{F}_{(j)}\right)$ which obey

$$
\xi_{(j)}^{2 N}=i d, \quad \xi_{(j)} \circ \theta_{(j)} \circ \xi_{(j)}=\theta_{(j)}, \quad \theta_{(j)}^{2}=\xi_{(j)}^{N} .
$$

As a first consequence of our claim we observe that it gives rise to the identifications

$$
\left(\operatorname{Bos}_{N}\right)^{\mathbb{Z}_{2}}=\operatorname{Orb}_{N} \quad \text { and } \quad\left(\mathrm{SL}_{2 N}\right)^{\mathbb{Z}_{2}}=\mathrm{SO}_{2 N}
$$

for a suitable $\mathbb{Z}_{2}$ group of automorphisms. This can be seen as follows. By the identities (5.3) we have $\xi_{(j)} \circ \theta_{(j)}\left(F_{(j)}\right)=\theta_{(j)} \circ \xi_{(j)}^{-1}\left(F_{(j)}\right)$ for all $F_{(j)} \in \mathfrak{F}_{(j)}$. As a consequence, $A_{(j)} \in$ $\left(\mathfrak{F}_{(j)}\right)^{\mathbb{Z}_{2 N}}$ implies that also $\theta_{(j)}\left(A_{(j)}\right) \in\left(\mathfrak{F}_{(j)}\right) \mathbb{Z}_{2 N}$, and hence there exist restrictions $\vartheta_{(j)}$ of $\theta_{(j)}$ to the $\mathbb{Z}_{2 N}$-invariant subalgebras $\operatorname{Bos}_{N}=\left(\mathfrak{F}_{(1)}\right) \mathbb{Z}_{2 N}$ and $\operatorname{SL}_{2 N}=\left(\mathfrak{F}_{(2)}\right)^{\mathbb{Z}_{2 N}}$, respectively. Now by definition the automorphism $\xi_{(j)}$ of $\mathfrak{F}_{(j)}$ restricts to the identity on $\left(\mathfrak{F}_{(j)}\right)^{\mathbb{Z}_{2 N}}$, and therefore the relations (5.3) imply that $\vartheta_{(j)}^{2}=i d$ for $j=1,2$. Thus $\vartheta_{(j)} \in \operatorname{Aut}\left(\left(\mathfrak{F}_{(j)}\right)^{\mathbb{Z}_{2 N}}\right)$ are in fact $\mathbb{Z}_{2}$-automorphisms. Put differently, the restrictions $\vartheta_{(j)}$ exist because $\mathbb{Z}_{2 N} \subset \mathrm{Q}_{N}$ is a normal subgroup, and they are $\mathbb{Z}_{2}$-automorphisms because $\mathrm{Q}_{N} / \mathbb{Z}_{2 N} \cong \mathbb{Z}_{2}$.

Now let us consider the automorphism

$$
\xi:=\xi_{(1)} \otimes \xi_{(2)} \in \operatorname{Aut}(\mathfrak{F}) \equiv \operatorname{Aut}\left(\mathfrak{F}_{(1)} \otimes \mathfrak{F}_{(2)}\right),
$$

which represents the generator $(x, x)$ of the diagonal subgroup $\mathbb{Z}_{2 N}^{\text {(diag) }} \subset \mathbb{Z}_{2 N} \times \mathbb{Z}_{2 N}$. The action of the full group $\mathbb{Z}_{2 N} \times \mathbb{Z}_{2 N}$ can be obtained by including another $\mathbb{Z}_{2 N}$-automorphism, say

$$
\tilde{\xi}:=i d \otimes \xi_{(2)},
$$

which realizes the element $(1, x)$ of $\mathbb{Z}_{2 N} \times \mathbb{Z}_{2 N}$. Now of course $\xi$ and $\tilde{\xi}$ commute (or in more mathematical terms, the diagonal subgroup $\mathbb{Z}_{2 N}^{\text {(diag) }} \subset \mathbb{Z}_{2 N} \times \mathbb{Z}_{2 N}$ is normal), so that there exists a restriction $\zeta$ of $\tilde{\xi} \in \operatorname{Aut}(\mathfrak{F})$ to $\mathfrak{F}^{\mathbb{Z}_{2 N} \text { (diag) }}$. We claim that when evaluated on the $\mathrm{CAR}$ algebra $\mathrm{CAR} \cong \mathfrak{F}^{\mathbb{Z}_{2 N(\mathrm{diag})}}$, this restriction coincides with the Bogoliubov automorphism $\zeta_{1}$ that was defined in formula (4.9):

$$
\zeta=\zeta_{1}: \quad b_{r}^{i} \mapsto \mathrm{e}^{\mathrm{i} \pi / N} b_{r}^{i}, \quad c_{r}^{i} \mapsto \mathrm{e}^{-\mathrm{i} \pi / N} c_{r}^{i} .
$$

8 Recall that in this isomorphism it is understood that the action of $\mathfrak{F}^{\mathbb{Z}_{2 N} \text { (diag) }}$ is restricted to the vacuum sector with respect to $\mathbb{Z}_{2 N}^{(\text {diag })}$. 
Next we also define $\theta:=\theta_{(1)} \otimes \theta_{(2)} \in \operatorname{Aut}\left(\mathfrak{F}_{(1)} \otimes \mathfrak{F}_{(2)}\right)$. Clearly, $\xi$ and $\theta$ generate the diagonal subgroup $\mathrm{Q}_{N}^{\text {(diag) }} \subset \mathrm{Q}_{N} \times \mathrm{Q}_{N}$. Now denote by $\vartheta$ the restriction of $\theta \in \operatorname{Aut}(\mathfrak{F})$ to $\mathfrak{F}^{\mathbb{Z}_{2 N} \text { (diag) }}$, which again exists because $\mathbb{Z}_{2 N}^{\text {(diag) }} \subset \mathrm{Q}_{N}^{\text {(diag) }}$ is normal. Since $\xi$ acts trivially on $\mathfrak{F}^{\mathbb{Z}_{2 N} \text { (diag), }}$, we learn that $\vartheta^{2}=$ id, i.e. $\vartheta$ generates a $\mathbb{Z}_{2}$ group. We claim that this $\mathbb{Z}_{2}$-action on $\mathrm{CAR} \cong \mathfrak{F}^{\mathbb{Z}_{2 N(\text { diag })}}$ is realized as the exchange

$$
\vartheta: \quad b_{r}^{i} \leftrightarrow c_{r}^{i}
$$

of the fermion modes.

We will verify our claims in the following analysis of characters. Moreover, we will see in section 6 that the $\vartheta$-invariant linear combinations of $\mathrm{SL}_{2 N}$-generators are precisely the generators of $\mathrm{SO}_{2 N}$, and that the $\vartheta$-invariant combinations of $\operatorname{Bos}_{N}$-generators are precisely the generators of $\operatorname{Orb}_{N}$. We start by noting that $\left[\mathrm{Q}_{N}: \mathbb{Z}_{2 N}\right]=2$. Correspondingly it is not difficult to construct the induction of the irreducible representations $\pi_{a}$ of $\mathbb{Z}_{2 N}$ to its extension $\mathrm{Q}_{N}$. Namely, for $0<a<N$ the irreducible $\mathbb{Z}_{2 N}$-representations $\pi_{a}$ and $\pi_{2 N-a}$ combine to a two-dimensional irreducible representation of $\mathrm{Q}_{N}$, while $\pi_{0}$ and $\pi_{N}$ split into the direct sum of two one-dimensional representations. Denoting the group dual (i.e., the set of irreducible characters) of the non-abelian group $\mathrm{Q}_{N}$ by

$$
\hat{\mathrm{Q}}_{N}=\{\mathrm{o}, \mathrm{v}, \mathrm{s}, \mathrm{c}\} \cup\{a \mid a=1,2, \ldots, N-1\},
$$

this is written as

$$
\begin{aligned}
& \operatorname{ind}_{\mathbb{Z}_{2 N}}^{\mathrm{Q}_{N}}\left(\pi_{a}\right)=\operatorname{ind}_{\mathbb{Z}_{2 N}}^{\mathrm{Q}_{N}}\left(\pi_{2 N-a}\right)=\pi_{a}^{\left(\mathrm{Q}_{N}\right)} \quad \text { for } 0<a<N, \\
& \operatorname{ind}_{\mathbb{Z}_{2 N}}^{\mathrm{Q}_{N}}\left(\pi_{0}\right)=\pi_{\circ}^{\left(\mathrm{Q}_{N}\right)} \oplus \pi_{\mathrm{v}}^{\left(\mathrm{Q}_{N}\right)}, \quad \operatorname{ind}_{\mathbb{Z}_{2 N}}^{\mathrm{Q}_{N}}\left(\pi_{N}\right)=\pi_{\mathrm{s}}^{\left(\mathrm{Q}_{N}\right)} \oplus \pi_{\mathrm{c}}^{\left(\mathrm{Q}_{N}\right)} .
\end{aligned}
$$

Next we study what happens when we extend the diagonal subgroup $\mathbb{Z}_{2 N}^{\text {(diag) }} \subset \mathbb{Z}_{2 N} \times \mathbb{Z}_{2 N}$ to the diagonal subgroup $\mathrm{Q}_{N}^{\text {(diag) }} \subset \mathrm{Q}_{N} \times \mathrm{Q}_{N}$. Applying the general result (2.7) to the situation described by (5.10), we learn that under the action of $\mathfrak{F}^{\mathrm{Q}_{N} \text { (diag) }} \subset \mathfrak{F}^{\mathbb{Z}_{2 N} \text { (diag) }}$ the fermionic Fock space $\mathcal{H}^{(\text {CAR })} \equiv \mathcal{H}_{0}$ splits as $\mathcal{H}_{0}=\mathcal{H}_{\circ} \oplus \mathcal{H}_{\mathrm{v}}$. Moreover, $\mathcal{H}_{\circ}=\mathcal{H}_{+}^{(\mathrm{CAR})}$ and $\mathcal{H}_{\mathrm{v}}=\mathcal{H}_{-}^{(\mathrm{CAR})}$ are the even and odd subspaces with respect to the $\mathbb{Z}_{2}$-automorphism $\vartheta$ of (5.8), respectively. When we further extend the gauge group to the full $\mathrm{Q}_{N} \times \mathrm{Q}_{N}$ group, these spaces further decompose into sectors of $\mathfrak{F}^{\mathrm{Q}_{N} \times \mathrm{Q}_{N}}=\left(\mathfrak{F}_{(\mathrm{Bos})}\right)^{\mathrm{Q}_{N}} \otimes\left(\mathfrak{F}_{(\mathrm{SL})}\right)^{\mathrm{Q}_{N}}$. Now the latter are of course tensor products of the sectors of $\left(\mathfrak{F}_{(\text {Bos })}\right)^{\mathrm{Q}_{N}}$ and $\left(\mathfrak{F}_{(\mathrm{SL})}\right)^{\mathrm{Q}_{N}}$, which we denote by $\mathcal{H}_{\alpha}^{(\mathrm{Bos})} \equiv \mathcal{H}_{\alpha}^{(\mathrm{Bos} ; 2 N)}$ and $\mathcal{H}_{\beta}^{(\mathrm{SL})} \equiv \mathcal{H}_{\beta}^{(\mathrm{SL} ; 2 N)}$, respectively. Implementing once again (2.7) as well as the formula (2.10), we then conclude that this decomposition reads

$$
\mathcal{H}_{+}^{(\mathrm{CAR})}=\bigoplus_{\alpha, \beta \in \hat{\mathrm{Q}}_{N}} \mathcal{H}_{\alpha}^{(\mathrm{Bos})} \otimes \mathcal{H}_{\beta}^{(\mathrm{SL})} \otimes \mathbb{C}^{N_{\alpha \beta}^{\circ}}, \quad \mathcal{H}_{-}^{(\mathrm{CAR})}=\bigoplus_{\alpha, \beta \in \hat{\mathrm{Q}}_{N}} \mathcal{H}_{\alpha}^{(\mathrm{Bos})} \otimes \mathcal{H}_{\beta}^{(\mathrm{SL})} \otimes \mathbb{C}^{N_{\alpha \beta}^{\mathrm{v}}}
$$


where $N_{\alpha \beta}^{\gamma}$ are the fusion coefficients of $\mathrm{Q}_{N}$, i.e. more explicitly,

$$
\begin{aligned}
& \mathcal{H}_{+}^{(\mathrm{CAR})}=\mathcal{H}_{\circ}^{(\mathrm{Bos})} \otimes \mathcal{H}_{\circ}^{(\mathrm{SL})} \oplus \mathcal{H}_{\mathrm{v}}^{(\mathrm{Bos})} \otimes \mathcal{H}_{\mathrm{v}}^{(\mathrm{SL})} \oplus \bigoplus_{\alpha=1}^{N-1} \mathcal{H}_{\alpha}^{(\mathrm{Bos})} \otimes \mathcal{H}_{\alpha}^{(\mathrm{SL})} \\
& \oplus \begin{cases}\mathcal{H}_{\mathrm{s}}^{(\mathrm{Bos})} \otimes \mathcal{H}_{\mathrm{s}}^{(\mathrm{SL})} \oplus \mathcal{H}_{\mathrm{c}}^{(\mathrm{Bos})} \otimes \mathcal{H}_{\mathrm{c}}^{(\mathrm{SL})} & \text { for } N \text { even, } \\
\mathcal{H}_{\mathrm{s}}^{(\mathrm{Bos})} \otimes \mathcal{H}_{\mathrm{c}}^{(\mathrm{SL})} \oplus \mathcal{H}_{\mathrm{c}}^{(\mathrm{Bos})} \otimes \mathcal{H}_{\mathrm{s}}^{(\mathrm{SL})} & \text { for } N \text { odd, }\end{cases} \\
& \mathcal{H}_{-}^{(\mathrm{CAR})}=\mathcal{H}_{\circ}^{(\mathrm{Bos})} \otimes \mathcal{H}_{\mathrm{v}}^{(\mathrm{SL})} \oplus \mathcal{H}_{\mathrm{v}}^{(\mathrm{Bos})} \otimes \mathcal{H}_{\circ}^{(\mathrm{SL})} \oplus \bigoplus_{\alpha=1}^{N-1} \mathcal{H}_{\alpha}^{(\mathrm{Bos})} \otimes \mathcal{H}_{\alpha}^{(\mathrm{SL})} \\
& \oplus \begin{cases}\mathcal{H}_{\mathrm{s}}^{(\mathrm{Bos})} \otimes \mathcal{H}_{\mathrm{c}}^{(\mathrm{SL})} \oplus \mathcal{H}_{\mathrm{c}}^{(\mathrm{Bos})} \otimes \mathcal{H}_{\mathrm{s}}^{(\mathrm{SL})} & \text { for } N \text { even, } \\
\mathcal{H}_{\mathrm{s}}^{(\mathrm{Bos})} \otimes \mathcal{H}_{\mathrm{s}}^{(\mathrm{SL})} \oplus \mathcal{H}_{\mathrm{c}}^{(\mathrm{Bos})} \otimes \mathcal{H}_{\mathrm{c}}^{(\mathrm{SL})} & \text { for } N \text { odd }\end{cases}
\end{aligned}
$$

On the other hand, it is not difficult to check - some details are provided in appendix B - that the characters of $\mathcal{H}_{+}^{(\mathrm{CAR})}$ and $\mathcal{H}_{-}^{\text {(CAR) }}$ (i.e. the characters of the irreducible modules of $\mathrm{CAR}^{\mathbb{Z}_{2}}$ ) can be decomposed as

$$
\begin{aligned}
& \chi_{+}^{\left[\mathbb{Z}_{2}\right]}=\chi_{\circ}^{(\mathrm{Orb})} \chi_{\circ}^{(\mathrm{SO})}+\chi_{\mathrm{v}}^{(\mathrm{Orb})} \chi_{\mathrm{v}}^{(\mathrm{SO})}+\chi_{\mathrm{s}}^{(\mathrm{Orb})} \chi_{\mathrm{s}}^{(\mathrm{SO})}+\chi_{\mathrm{c}}^{(\mathrm{Orb})} \chi_{\mathrm{c}}^{(\mathrm{SO})}+\sum_{\alpha=1}^{N-1} \chi_{\alpha}^{(\mathrm{Orb})} \chi_{\alpha}^{(\mathrm{sO})}, \\
& \chi_{-}^{\left[\mathbb{Z}_{2}\right]}=\chi_{\circ}^{(\mathrm{Orb})} \chi_{\mathrm{v}}^{(\mathrm{SO})}+\chi_{\mathrm{v}}^{(\mathrm{Orb})} \chi_{\circ}^{(\mathrm{SO})}+\chi_{\mathrm{s}}^{(\mathrm{Orb})} \chi_{\mathrm{s}}^{(\mathrm{SO})}+\chi_{\mathrm{c}}^{(\mathrm{Orb})} \chi_{\mathrm{c}}^{(\mathrm{SO})}+\sum_{\alpha=1}^{N-1} \chi_{\alpha}^{(\mathrm{Orb})} \chi_{\alpha}^{(\mathrm{SO})}
\end{aligned}
$$

into products of irreducible characters $\chi^{(\text {Orb })}$ of the $c=1$ orbifold theory Orb $_{N}$ and of irreducible characters $\chi^{(\mathrm{so})}$ of the level two $\widehat{\mathfrak{s o}}(2 N)$ WZW theory. Now comparing the formulae (5.12) and (5.13) (and recalling that $\chi_{\mathrm{s}}^{(\mathrm{Orb})}=\chi_{\mathrm{c}}^{(\mathrm{Orb})}$ as well as $\chi_{\mathrm{s}}^{(\mathrm{so})}=\chi_{\mathrm{c}}^{(\mathrm{so})}$ ) leads us to the conclusion that the sectors of the gauge invariant algebras $\left(\mathfrak{F}_{(\text {Boss }}\right)^{Q_{N}}$ and $\left(\mathfrak{F}_{(\mathrm{SL})}\right)^{Q_{N}}$ indeed coincide with those of $\mathrm{Orb}_{N}$ and $\mathrm{SO}_{2 N}$, respectively. This finally reproduces the statement of our claim.

We further support our claim by the following consideration. Recall that $\vartheta \in \operatorname{Aut}\left(\mathfrak{F}^{\mathbb{Z}_{2 N}}\right)$ satisfies $\vartheta^{2}=$ id. This implies that $\zeta$ and $\vartheta$ fulfill

$$
\zeta^{2 N}=\mathrm{id}, \quad \zeta \circ \vartheta \circ \zeta=\vartheta, \quad \vartheta^{2}=\mathrm{id}
$$

Thus they provide a representation of the dihedral group $\mathrm{D}_{2 N}=\mathbb{Z}_{2 N} \times \mathbb{Z}_{2}$ by automorphisms of $\mathfrak{F}^{\mathbb{Z}_{2 N} \text { (diag) }}$. We can therefore consider the algebra $\left(\mathfrak{F}^{\mathbb{Z}_{2 N}}\right)^{\mathrm{D}_{2 N}}$, which is the invariant part of $\mathfrak{F}^{\mathbb{Z}_{2 N} \text { (diag) }}$ with respect to the automorphisms $\tilde{\xi}$ and $\theta$, or what is the same, the invariant part of $\mathfrak{F}$ with respect to $\xi, \tilde{\xi}$ and $\theta$. Now $\xi, \tilde{\xi}$ and $\theta$ provide a representation of a subgroup $K_{N} \subset \mathrm{Q}_{N} \times \mathrm{Q}_{N}$ that is obtained by adjoining the diagonal generator $(y, y)$ to $\mathbb{Z}_{2 N} \times \mathbb{Z}_{2 N}$. Note that $\left[\mathrm{Q}_{N} \times \mathrm{Q}_{N}: K_{N}\right]=2$ and that the diagonal subgroup $\mathbb{Z}_{2 N}^{\text {(diag) }} \subset K_{N}$ is normal. Now $K_{N} / \mathbb{Z}_{2 N}^{\text {(diag) }} \cong \mathrm{D}_{2 N}$, and this is the reason why the restrictions $\zeta$ and $\vartheta$ of $\tilde{\xi}$ and $\theta$ provide a representation of $D_{2 N}$ in $\operatorname{Aut}\left(\mathfrak{F}^{\mathbb{Z}_{2 N}}\right)$. We conclude that

$$
\left(\mathfrak{F}_{(\mathrm{Bos} ; 2 N)} \otimes \mathfrak{F}_{(\mathrm{SL} ; 2 N)}\right)^{\mathrm{Q}_{N} \times \mathrm{Q}_{N}} \subset \mathfrak{F}^{K_{N}} \equiv\left(\mathfrak{F}^{\mathbb{Z}_{2 N}}\right)^{\mathrm{D}_{2 N}}
$$

or in other words,

$$
\mathrm{Orb}_{N} \otimes \mathrm{SO}_{2 N} \subset \mathrm{CAR}^{\mathrm{D}_{2 N}}
$$


As it is a rather tedious calculation, we refrain from applying the whole machinery of section 2 to the (gauge) subgroup $K_{N}=H \subset G=\mathrm{Q}_{N} \times \mathrm{Q}_{N}$. Rather, we restrict the discussion to confirming the validity of the $\mathbb{Z}_{2}$-type inclusion (5.16), which is achieved by the following argument. Under the action of $\mathrm{CAR}^{\mathrm{D}_{2 N}} \cong \mathfrak{F}^{K_{N}}$ the vacuum sector $\mathcal{H}_{0}=\mathcal{H}^{\text {(CAR) }}$ of $\mathfrak{F}^{\mathbb{Z}_{2 N} \text { (diag) }}$ splits into sectors labelled by $\hat{D}_{2 N}$, and as the inclusion (5.16) is of $\mathbb{Z}_{2}$-type, in this process each sector can split into at most two $\mathfrak{F}^{\mathrm{Q}_{N} \times \mathrm{Q}_{N}}$-sectors. Now the group $\mathrm{D}_{2 N}$ is precisely represented in Aut(CAR) by the automorphisms (5.7) and (5.8), while for the characters of $\mathrm{CAR}^{\mathrm{D}_{2 N}}$ we obtain

$$
\begin{array}{ll}
\chi_{\circ}^{\left[\mathrm{D}_{2 N}\right]}=\chi_{\circ}^{(\mathrm{Orb})} \chi_{\circ}^{(\mathrm{SO})}+\chi_{\mathrm{v}}^{(\mathrm{Orb})} \chi_{\mathrm{v}}^{(\mathrm{SO})}, & \chi_{\mathrm{v}}^{\left[\mathrm{D}_{2 N}\right]}=\chi_{\circ}^{(\mathrm{Orb})} \chi_{\mathrm{v}}^{(\mathrm{SO})}+\chi_{\mathrm{v}}^{(\mathrm{Orb})} \chi_{\circ}^{(\mathrm{SO})}, \\
\chi_{\mathrm{s}}^{\left[\mathrm{D}_{2 N}\right]}=\chi_{\mathrm{s}}^{(\mathrm{Orb})} \chi_{\mathrm{s}}^{(\mathrm{SO})}+\chi_{\mathrm{c}}^{(\mathrm{Orb})} \chi_{\mathrm{c}}^{(\mathrm{SO})}, & \chi_{\mathrm{c}}^{\left[\mathrm{D}_{2 N}\right]}=\chi_{\mathrm{s}}^{(\mathrm{Orb})} \chi_{\mathrm{s}}^{(\mathrm{SO})}+\chi_{\mathrm{c}}^{(\mathrm{Orb})} \chi_{\mathrm{c}}^{(\mathrm{SO})}, \\
\chi_{\alpha}^{\left[\mathrm{D}_{2 N}\right]}=\chi_{\alpha}^{(\mathrm{Orb})} \chi_{\alpha}^{(\mathrm{SO})} \quad \text { for } \alpha \in\{1,2, \ldots, N-1\} .
\end{array}
$$

(for more details, see appendix B). Again we conclude by comparison that (5.17) precisely corresponds to the decomposition of $\mathrm{CAR}^{\mathrm{D}_{2 N}}$-sectors into tensor products of the sectors of $\mathrm{Orb}_{N}$ and $\mathrm{SO}_{2 N} \cdot$.

The above arguments in favor of our claims are certainly not rigorous, because we employ the Virasoro-specialized characters which do not encode the complete structure of the respective vector spaces. Nevertheless our claims are bound to be correct. For instance, it would otherwise be a complete mystery why relations among characters of the type derived above should be valid. Note in particular that our formulae hold simultaneously for all values of the integer $N$. Moreover, the quite different arguments that we will present in the following section provide further support to our claims.

\section{Fourier modes of the observables}

In this section we express the Fourier modes of the observables for the various models of our interest through the Fourier modes of the free fermions. This will in particular allow us to confirm various statements made earlier from a different point of view.

We first need to define a normal ordering of bilinears of fermion modes. We adopt the convention that the symbol $a_{r}^{i}(r \in \mathbb{Z}+1 / 2, i \in\{1,2, \ldots, 2 N\})$, stands for either of the Fourier modes $b_{r}^{i}$ or $c_{r}^{i}$ of the fermions. Our normal ordering prescription then reads

$$
: a_{r}^{i} a_{s}^{j}::= \begin{cases}a_{r}^{i} a_{s}^{j} & \text { for } s>0, \\ -a_{s}^{j} a_{r}^{i} & \text { for } s<0 .\end{cases}
$$

One now checks by direct computation that the combinations

$$
J_{m}^{i j}:=\sum_{r \in \mathbb{Z}+1 / 2}: b_{r}^{i} c_{m-r}^{j}:
$$

${ }^{9}$ Another indirect confirmation of our claim follows from the following observation. In the space $\mathcal{H}^{\text {(CAR) }}$ there must be simultaneous highest weight vectors of $\mathrm{Orb}_{N}$ and $\mathrm{SO}_{2 N}$ which correspond to the blocks that appear in the decomposition (5.17). In [9] we determined the larger set of simultaneous highest weight vectors of $\mathrm{SO}_{2 N}$ and the orbifold Virasoro algebra. Now inspecting the orbifold conformal weights of those vectors one learns that the simultaneous highest weight vectors of $\mathrm{Orb}_{N}$ and $\mathrm{SO}_{2 N}$ are given by the zero grade $(n=0)$ vectors among those in Eqs. (8.8), (8.11), (8.12), (8.13) and also (10.12) and (10.13) of [9]. One can check that the $\mathrm{D}_{2 N}$ transformation properties of these vectors are indeed in agreement with the sector decomposition (5.17). 
with $i, j \in\{1,2, \ldots, 2 N\}$ and $m \in \mathbb{Z}$ satisfy the commutation relations

$$
\left[J_{m}^{i j}, J_{n}^{k l}\right]=\delta_{j k} J_{m+n}^{i l}-\delta_{i l} J_{m+n}^{k j}+m \delta_{j k} \delta_{i l} \delta_{m+n, 0} \mathbf{1}
$$

and hence span a level one $\widehat{\mathfrak{g l}}(2 N)$ current algebra. More precisely, this Lie algebra is the direct sum of a level one $\widehat{\mathfrak{s l}}(2 N)$ affine Kac-Moody algebra and a $\widehat{\mathfrak{u}}(1)$ current algebra. The generators of the level one $\widehat{\mathfrak{s l}}(2 N)$ algebra consist of the linear combinations

$$
H_{m}^{i}:=J_{m}^{i i}-J_{m}^{i+1 i+1} \quad \text { for } i=1,2, \ldots, 2 N-1
$$

and of

$$
E_{m}^{i j}:=J_{m}^{i j} \quad \text { for } i \neq j .
$$

The zero modes $(m=0)$ generate a subalgebra isomorphic to the simple Lie algebra $\mathfrak{s l}(2 N)$. In particular, the modes $H_{0}^{i}$ span the Cartan subalgebra of $\mathfrak{s l}(2 N)$, and for $i<j$ the $E_{0}^{i j}$ constitute the raising operators of $\mathfrak{s l}(2 N)$, corresponding to the positive $\mathfrak{s l}(2 N)$-roots $\alpha_{i j} \equiv \alpha_{(i)}+\alpha_{(i+1)}+\cdots+\alpha_{(j-2)}+\alpha_{(j-1)}$ (where $\alpha_{(k)}$ denote the simple roots of $\mathfrak{s l}(2 N)$ ), while for $j<i$ they are lowering operators, corresponding to the negative $\mathfrak{s l}(2 N)$-roots $-\alpha_{j i}$. The $\widehat{\mathfrak{u}}(1)$ current algebra is spanned by

$$
J_{m}:=\frac{1}{\sqrt{2 N}} \sum_{i=1}^{2 N} J_{m}^{i i}
$$

with relations

$$
\left[J_{m}, J_{n}\right]=\delta_{m+n, 0} \mathbf{1} \text {. }
$$

The *-operation acts on the currents (6.2) as $\left(J_{m}^{i j}\right)^{*}=J_{-m}^{j i}$, and their commutation relations with the fermion modes read

$$
\left[J_{m}^{i j}, b_{r}^{k}\right]=\delta^{j, k} b_{m+r}^{i}, \quad\left[J_{m}^{i j}, c_{r}^{k}\right]=-\delta^{i, k} c_{m+r}^{j},
$$

so that in particular

$$
\left[J_{m}, b_{r}^{i}\right]=\frac{1}{\sqrt{2 N}} b_{m+r}^{i}, \quad\left[J_{m}, c_{r}^{i}\right]=-\frac{1}{\sqrt{2 N}} c_{m+r}^{i} .
$$

In addition to the current algebra, the free fermions also bring along a Virasoro algebra, with generators

$$
L_{m}^{(\mathrm{CAR})}=-\frac{1}{2} \sum_{r \in \mathbb{Z}+1 / 2} \sum_{i=1}^{2 N}\left(r-\frac{m}{2}\right): b_{r}^{i} c_{m-r}^{i}:
$$

Let us now interpret these observations from the perspective of sections 3 and 4 . The (unbounded) observables of the $\mathfrak{s l}(2 N)$ level one WZW theory are well known [8]. They consist precisely of the $\widehat{\mathfrak{s l}}(2 N)$ currents with modes (6.4) and (6.5), together with fields that are obtainable from the currents by taking derivatives and forming normalordered products. Among the latter there is in particular the associated Sugawara energymomentum tensor, the Fourier modes of which are given by the affine Sugawara formula

$$
L_{m}^{(\mathrm{SL})}=\sum_{n \in \mathbb{Z}}\left(\sum_{\substack{i, j=1 \\ i<j}}^{2 N}: J_{n}^{i j} J_{m-n}^{j i}:+\frac{1}{2} \sum_{i, j=1}^{2 N} G_{i j}: H_{n}^{i} H_{m-n}^{j}:\right)
$$


$\left(G_{i j}\right.$ denotes the inverse of the Cartan matrix of $\mathfrak{s l}(2 N)$, and the normal ordering prescription is similar to the one in (6.1)), and hence they lie in a suitable completion of the universal enveloping algebra of the current modes.

It follows that in order to be in agreement with the isomorphism (4.3), the observable algebras $\operatorname{Bos}_{N}(I)$ of the $c=1$ theory are given by the commutants of $\operatorname{SL}_{2 N}$ in $\operatorname{CAR}(I)^{\mathbb{Z}_{2 N}}$. This certainly includes the bounded local functions of the $\widehat{\mathfrak{u}}(1)$ current $J \mathbb{P}$ as well as those of the associated energy-momentum tensor whose modes are

$$
L_{m}^{(\text {Bos })}=\frac{1}{2} \sum_{n \in \mathbb{Z}}: J_{n} J_{m-n}:
$$

(By carefully treating multiple normal orderings of the fermion modes, one can check that $L_{m}^{(\text {Bos })}+L_{m}^{(\mathrm{SL})}=L_{m}^{(\mathrm{CAR})}$.) We are now looking for further unbounded observables associated to the $\operatorname{Bos}_{N}$ theory. In particular, we would like to find expressions that commute with the $\widehat{\mathfrak{s l}}(2 N)$ modes and can be interpreted as the Fourier modes of fields $\phi$ that have integral conformal weight $\Delta(\phi)$ (with respect to $L_{0}^{(\mathrm{CAR})}$ ) and that can play the rôle of primary conformal fields in the sense of [14. Then the associated local bounded functions should be elements of $\operatorname{Bos}_{N}$. Taking into account the isomorphism (4.3), we have to expect that these modes are $\mathbb{Z}_{2 N}$-invariant infinite series of normal-ordered products of fermion modes; moreover, we in fact need only to consider normal-ordered multilinears which are summed over like

$$
\phi_{m}^{i_{1} i_{2} \ldots i_{p}}=\sum_{\substack{r_{1}, r_{2}, \ldots, r_{p-1} \\ \in \mathbb{Z}+1 / 2}}: a_{r_{1}}^{i_{1}} a_{r_{2}}^{i_{2}} \cdots a_{r_{p-1}}^{i_{p-1}} a_{m-r_{1}-r_{2}-\ldots-r_{p-1}}^{i_{p}}:
$$

with $p=2 \Delta(\phi)$ even. In addition, we can focus our attention to a small subset of these unbounded observables, namely to those from which all others can be obtained by the operations of taking derivatives and of forming normal-ordered products; for brevity, we will refer to this subset as the basic (unbounded) observables.

Now by a suitable relabelling of the summation indices on the right-hand side of (6.13) we deduce from the anticommutativity of the $b$ respectively the $c$ modes among themselves that we can assume that equality $i_{k}=i_{l}$ for $k \neq l$ appears only if one is dealing with two different types of modes, i.e. only if $a_{r_{k}}^{i_{k}}=b_{r_{k}}^{i_{k}}$ and $a_{r_{l}}^{i_{l}}=c_{r_{l}}^{i_{l}}$ (or the other way round). Employing the basic commutation relations (6.8) to compute the commutator of $\phi_{m}^{i_{1} i_{2} \ldots i_{p}}$ with $J_{n}^{j k}$ one then finds the following. First note that expressions which are neutral with respect to the gauge group $\mathbb{Z}_{2 N}(4.9)$ must involve products for which the numbers of $b$ and $c$ factors differ by a multiple of $2 N$. Now when (6.13) contains an equal number of $b$ and $c$ modes, then it commutes with the $\widehat{\mathfrak{s l}}(2 N)$ currents precisely if it is a normal-ordered product of the Fourier modes of the $\widehat{\mathfrak{u}}(1)$ current $J$ (i.e. the $J_{m}$ (6.6)) and/or its derivatives. A similar analysis shows that for any other $\mathbb{Z}_{2 N}$-neutral combination $X$ to commute with the $\widehat{\mathfrak{s l}}(2 N)$ currents it is necessary and sufficient that $X$ is obtainable by taking derivatives and/or forming normal-ordered products of the $\widehat{\mathfrak{u}}(1)$

\footnotetext{
10 Thus in particular the currents associated to $\mathfrak{F}_{(\mathrm{Bos})} \otimes \mathfrak{F}_{(\mathrm{SL})}$ are represented in the form of a tensor product, i.e. the $\widehat{\mathfrak{u}}(1)$ current acts as $J \otimes \mathbf{1}$ while the $\widehat{\mathfrak{s l}}(2 N)$ currents act as $\mathbf{1} \otimes H_{m}^{i}$ and $\mathbf{1} \otimes E_{m}^{i j}$.
} 
current modes and of the modes

$$
\begin{aligned}
B_{m} & :=\sum_{\substack{r_{1}, r_{2}, \ldots, r_{2 N-1} \\
\in \mathbb{Z}+1 / 2}} b_{r_{1}}^{1} b_{r_{2}}^{2} \cdots b_{r_{2 N-1}}^{2 N-1} b_{m-r_{1}-r_{2}-\ldots-r_{2 N-1}}^{2 N}, \\
C_{m}:= & \sum_{\substack{r_{1}, r_{2}, \ldots, r_{2 N-1} \\
\in \mathbb{Z}+1 / 2}} c_{r_{1}}^{1} c_{r_{2}}^{2} \cdots c_{r_{2 N-1}}^{2 N-1} c_{m-r_{1}-r_{2}-\ldots-r_{2 N-1}}^{2 N} .
\end{aligned}
$$

One can check that the point-like localized fields which have (6.14) as their Fourier modes, namely $B(z):=\sum_{m \in \mathbb{Z}} z^{m-N} B_{m}=b^{1}(z) b^{2}(z) \cdots b^{2 N}(z)$ and $C(z):=\sum_{m \in \mathbb{Z}} z^{m-N} C_{m}=c^{1}(z)$ $c^{2}(z) \cdots c^{2 N}(z)$, are primary conformal fields and have conformal weight $\Delta(B)=\Delta(C)=N$, both with respect to the fermion energy-momentum tensor $(6.10)$ and with respect to the $c=1$ energy-momentum tensor (6.12) (which is compatible because they commute with the $\widehat{\mathfrak{s l}}(2 N)$ current algebra and hence with its energy-momentum tensor).

We conclude that the basic observables of the $c=1$ theory consist of the $\widehat{\mathfrak{u}}(1)$ current and the fields $B$ and $C$, which are related by charge conjugation. This is in complete agreement with the description of the observables that was given in [12]. The commutation relations of the modes (6.14) are of the form

$$
\begin{aligned}
& {\left[B_{m}, B_{n}\right]=0=\left[C_{m}, C_{n}\right],} \\
& {\left[B_{m}, C_{n}\right]=\mathrm{p}_{1} \delta_{m+n, 0} \mathbf{1}-\mathrm{p}_{2} \tilde{J}_{m+n}+\frac{1}{2} \mathrm{p}_{3}\left(: \tilde{J}^{2}:-\partial \tilde{J}\right)_{m+n}+\ldots,}
\end{aligned}
$$

where

$$
\mathrm{p}_{j} \equiv \mathrm{p}_{m, N ; j}:=\frac{1}{(2 N-j) !} \sum_{\ell=1}^{2 N-j}(m-N+j+\ell-1)
$$

for $j \in \mathbb{N}$ and

$$
\tilde{J}_{m}:=\sqrt{2 N} J_{m},
$$

and where the ellipsis stands for further terms involving $j$-fold normal-ordered products of the currents (combined with terms involving derivatives, in a similar way as for $: \tilde{J}^{2}:-\partial \tilde{J}$ ) and coefficients whose $m$ - and $N$-dependence takes the form of $\mathrm{p}_{j}$, for $j=4,5, \ldots, 2 N-1$.

Note that for $N=1$, the relations (6.15) amount to the statement that besides the $\widehat{\mathfrak{s l}}(2)$ affine Lie algebra with generators $(6.4)$ and $(6.5)$, i.e.

$$
\begin{aligned}
& J_{m}^{+}:=E_{m}^{12}=\sum_{r \in \mathbb{Z}+1 / 2}: b_{r}^{1} c_{m-r}^{2}:, \quad J_{m}^{-}:=E_{m}^{21}=\sum_{r \in \mathbb{Z}+1 / 2}: b_{r}^{2} c_{m-r}^{1}: \\
& J_{m}^{0}:=H_{m}^{1}=\sum_{r \in \mathbb{Z}+1 / 2}\left(: b_{r}^{1} c_{m-r}^{1}:-: b_{r}^{2} c_{m-r}^{2}:\right)
\end{aligned}
$$

another (relatively commuting) level one $\widehat{\mathfrak{s l}}(2)$ algebra is present, namely the one generated by

$$
\begin{aligned}
& K_{m}^{+}:=\mathrm{i} B_{m}=\mathrm{i} \sum_{r \in \mathbb{Z}+1 / 2}: b_{r}^{1} b_{m-r}^{2}:, \quad K_{m}^{-}:=\mathrm{i} C_{m}=\mathrm{i} \sum_{r \in \mathbb{Z}+1 / 2}: c_{r}^{1} c_{m-r}^{2}: \\
& K_{m}^{0}:=\sqrt{2} J_{m}=\sum_{r \in \mathbb{Z}+1 / 2}\left(: b_{r}^{1} c_{m-r}^{1}:+: b_{r}^{2} c_{m-r}^{2}:\right) .
\end{aligned}
$$

\footnotetext{
11 The summations ensure locality. Also note that owing to $\left[b_{r}^{i}, b_{s}^{j}\right]=0=\left[c_{r}^{i}, c_{s}^{j}\right]$ no normal ordering is
} required here. 
(In a different context, this has also been observed in [15].)

Next, let us study the observables which stay fixed under $\mathrm{Q}_{N}$, i.e. those which are the observables of the orbifold and $\widehat{\mathfrak{s o}}(2 N)$ theories. According to the results of section 5, we need to implement the additional $\mathbb{Z}_{2}$ transformation which operates on the fermion modes as the exchange $\vartheta(5.8)$. We first note that $\vartheta$ acts on the $\widehat{\mathfrak{g l}}(2 N)$ current modes (6.2) as

$$
\vartheta\left(J_{m}^{i j}\right)=-J_{m}^{j i}
$$

so that the invariant combinations are

$$
I_{m}^{i j}:=J_{m}^{i j}-J_{m}^{j i}
$$

for $m \in \mathbb{Z}$ and $i, j \in\{1,2, \ldots, 2 N\}$ with $i<j$. It follows from the commutation relations (6.3) that these modes span a level two $\widehat{\mathfrak{s o}}(2 N)$ affine Kac-Moody algebra. This is also easily understood by realizing that the real and imaginary parts $u_{r}^{i}$ and $v_{r}^{i}$ of the fermion modes, defined by

$$
b_{r}^{i}:=\frac{1}{\sqrt{2}}\left(u_{r}^{i}+\mathrm{i} v_{r}^{i}\right), \quad c_{r}^{i}:=\frac{1}{\sqrt{2}}\left(u_{r}^{i}-\mathrm{i} v_{r}^{i}\right),
$$

which satisfy $\vartheta\left(u_{r}^{i}\right)=u_{r}^{i}$ and $\vartheta\left(v_{r}^{i}\right)=-v_{r}^{i}$, constitute the Fourier modes of a two sets of real free fermions. Each of these realizes a real CAR algebra, and they mutually anticommute. The combinations (6.21) are expressed through these fermion modes by

$$
\begin{aligned}
I_{m}^{i j} & =\frac{1}{2} \sum_{r \in \mathbb{Z}+1 / 2}\left(: u_{r}^{i} u_{m-r}^{j}:-: u_{r}^{j} u_{m-r}^{i}:+: v_{r}^{i} v_{m-r}^{j}:-: v_{r}^{j} v_{m-r}^{i}:\right) \\
& =\sum_{r \in \mathbb{Z}+1 / 2}\left(: u_{r}^{i} u_{m-r}^{j}:+: v_{r}^{i} v_{m-r}^{j}:\right) .
\end{aligned}
$$

It then follows immediately (compare e.g. [9]) that we are indeed dealing with a level two $\widehat{\mathfrak{s o}}(2 N)$ affine Lie algebra.

Now we use again the knowledge that the basic observables of a WZW theory are given by the currents. From the inclusion (5.16) we therefore conclude that the observables $\mathrm{Orb}_{N}$ of the orbifold theory are contained in the commutant of the current algebra in the algebra $\mathrm{CAR}^{\mathrm{D}_{2 N}}$. As this is a proper inclusion, we cannot completely determine the orbifold observables this way. Nevertheless we can make a few observations which can be compared to the literature. First, the $\widehat{\mathfrak{u}}(1)$ current modes (6.6), which in terms of the real fermions read $J_{m}=\mathrm{i}(2 N)^{-1 / 2} \sum_{r \in \mathbb{Z}+1 / 2} \sum_{i=1}^{2 N} v_{r}^{i} u_{m-r}^{i}$, transform as $\vartheta\left(J_{m}\right)=-J_{m}$ and hence definitely do not belong to $\mathrm{Orb}_{N}$. Also, among the linear combinations of the $c=1$ observables (6.14), only $B_{m}+C_{m}$ are $\vartheta$-invariant; comparison with the results of [7] shows that they belong indeed to the basic unbounded observables of the orbifold theory, and hence the corresponding bounded functions are elements of $\operatorname{Orb}_{N}$. However, there are still further basic observables. These include in particular the energy-momentum tensor, whose modes can be written as

$$
L_{m}^{(\mathrm{Orb})}=L_{m}^{(\mathrm{CAR})}-L_{m}^{(\mathrm{SO})}
$$

(analogously to $L_{m}^{(\mathrm{Bos})}=L_{m}^{(\mathrm{CAR})}-L_{m}^{(\mathrm{SL})}$ ), where $L_{m}^{(\mathrm{SO})}$ is obtained from the $\widehat{\mathfrak{s o}}(2 N)$ currents (6.21) by the Sugawara formula. In terms of the fermion modes, this is a normal-ordered product which contains the fermion modes only in the quadratic form $u_{r}^{i} u_{s}^{i}$ and $v_{r}^{j} v_{s}^{j}$. 
According to [7] there is one other primary conformal field which is a basic observable, namely the combination $: J^{4}:-2: J \partial^{2} J:+3 / 2:(\partial J)^{2}:$.

In the special case $N=1$ the current algebra $\widehat{\mathfrak{s o}}(2 N)$ degenerates to $\widehat{\mathfrak{u}}(1)$, with modes $I_{m}^{12}=\sum_{r \in \mathbb{Z}+1 / 2}\left(: u_{r}^{1} u_{m-r}^{2}:+: v_{r}^{1} v_{m-r}^{2}:\right)$, or in terms of the $\widehat{\mathfrak{s l}}(2)$ modes $(6.18), I_{m}^{12}=J_{m}^{+}-$ $J_{m}^{-}$. Similarly, the orbifold observables $B_{m}+C_{m}$ become $B_{m}+C_{m}=-\mathrm{i}\left(K_{m}^{+}+K_{m}^{-}\right)=\sum_{r \in \mathbb{Z}+1 / 2}$ $\left(: u_{r}^{1} u_{m-r}^{2}:-: v_{r}^{1} v_{m-r}^{2}:\right)$, which again generate a $\widehat{\mathfrak{u}}(1)$ current algebra. In fact, in this case we are dealing with the tensor product of two $c=1$ circle theories, each correponding to the value $N_{\text {(Bos) }}=4$ of the integer that labels the circle theories.

Finally we note that by construction the real fermions $u^{i}$ and $v^{i}$ are of Neveu-Schwarz type. In terms of the orbifold and $\widehat{\mathfrak{s o}}(2 N)$ theories, this corresponds to the fact that we are dealing with untwisted sectors only. In order to investigate the twisted sectors as well, one would have to include also real fermions of Ramond type. Since for generic $N$ the twisted sectors have non-integral statistical dimension, they are not covered by the conventional DHR formalism, and hence are definitely beyond the scope of our present paper. On the other hand, when $N$ is a square number, then the statistical dimension of the twisted sectors is integral, and correspondingly an interpretation in terms of a DHR gauge group might again exist. At present we do not know of such an interpretation. But it is easy to see that such a gauge group would have to be an extension of $\mathrm{Q}_{N}$ by $\mathbb{Z}_{2}$. Moreover, it is likely that this extension should be central, in such a way that the twisted sectors can be interpreted as projective representations of the factor group $\mathrm{Q}_{N}$. (For $N=1$, this possibility is realized rather trivially as the extension from $\mathrm{Q}_{1} \equiv \mathbb{Z}_{4}$ to $\left.\mathbb{Z}_{8} \cdot\right)$

\section{A The finite groups $\mathrm{Q}_{N}$ and $\mathrm{D}_{2 N}$}

For any positive integer $N$, the generalized quaternion group $\mathrm{Q}_{N}$ is by definition the discrete group that is generated freely by elements $x$ and $y$ modulo the relations

$$
x^{2 N}=1, \quad x y x=y, \quad y^{2}=x^{N} .
$$

This is a finite group of order $\left|\mathrm{Q}_{N}\right|=4 N$. It has four one-dimensional representations $\pi_{\mathrm{o}}, \pi_{\mathrm{v}}, \pi_{\mathrm{s}}, \pi_{\mathrm{c}}$, as well as $N-1$ two-dimensional representations $\pi_{m}$ with representing matrices [16]

$$
\pi_{m}(x)=\left(\begin{array}{cc}
\mathrm{e}^{\mathrm{i} \pi m / N} & 0 \\
0 & \mathrm{e}^{-\mathrm{i} \pi m / N}
\end{array}\right), \quad \pi_{m}(y)=\left(\begin{array}{cc}
0 & (-1)^{m} \\
1 & 0
\end{array}\right) .
$$

The conjugacy classes and characters of $\mathrm{Q}_{N}$ are displayed in table 1. From the character table it follows in particular that the subring of the representation ring that is furnished by the one-dimensional representations is the group ring of $\mathbb{Z}_{2} \times \mathbb{Z}_{2}$ when $N$ is even, and the group ring of $\mathbb{Z}_{4}$ when $N$ is odd.

The cyclic group $\mathbb{Z}_{2 N}$ generated by $x$ is a normal subgroup of $\mathrm{Q}_{N} ; \mathrm{Q}_{N}$ is a non-split extension of this normal subgroup by $\mathbb{Z}_{2}$. It is illustrative to compare $\mathrm{Q}_{N}$ to the dihedral group $\mathrm{D}_{2 N}$ which is a split extension of its normal subgroup $\mathbb{Z}_{2 N}$ by $\mathbb{Z}_{2}$ and hence a semidirect product. $\mathrm{D}_{2 N}$ is by definition generated by elements $\tilde{x}, \tilde{y}$ subject to the relations

$$
\tilde{x}^{2 N}=1, \quad \tilde{x} \tilde{y} \tilde{x}=\tilde{y}, \quad \tilde{y}^{2}=1 .
$$

12 The first of these relations is not independent. We keep it to demonstrate the similarity with (A.3) below. 
Table 1: Character table for $\mathrm{Q}_{N}$

\begin{tabular}{|c||c|c|c|c|c|}
\hline class & $\{1\}$ & $\begin{array}{c}\left\{x^{k}, x^{-k}\right\} \\
(k \in\{1,2, \ldots, N-1\})\end{array}$ & $\left\{x^{N}\right\}$ & $\begin{array}{c}\left\{y x^{2 l} \mid\right. \\
l=0,1, \ldots, N-1\}\end{array}$ & $\begin{array}{c}\left\{y x^{2 l+1} \mid\right. \\
l=0,1, \ldots, N-1\}\end{array}$ \\
\hline \hline$\chi_{\circ}$ & 1 & 1 & 1 & 1 & 1 \\
$\chi_{\mathrm{v}}$ & 1 & 1 & 1 & -1 & -1 \\
$\chi_{\mathrm{s}}$ & 1 & $(-1)^{k}$ & $(-1)^{N}$ & $\mathrm{i}^{N}$ & $-\mathrm{i}^{N}$ \\
$\chi_{\mathrm{c}}$ & 1 & $(-1)^{k}$ & $(-1)^{N}$ & $-\mathrm{i}^{N}$ & $\mathrm{i}^{N}$ \\
$\chi_{m}$ & 2 & $2 \cos (\pi m k / N)$ & $2(-1)^{m}$ & 0 & 0 \\
\hline
\end{tabular}

We have $\left|\mathrm{D}_{2 N}\right|=4 N$, and there are four one-dimensional representations $\tilde{\pi}_{\mathrm{o}}, \tilde{\pi}_{\mathrm{v}}, \tilde{\pi}_{\mathrm{s}}, \tilde{\pi}_{\mathrm{c}}$, and $N-1$ two-dimensional representations $\tilde{\pi}_{m}$ with matrices

$$
\tilde{\pi}_{m}(\tilde{x})=\left(\begin{array}{cc}
\mathrm{e}^{\mathrm{i} \pi m / N} & 0 \\
0 & \mathrm{e}^{-\mathrm{i} \pi m / N}
\end{array}\right), \quad \quad \tilde{\pi}_{m}(\tilde{y})=\left(\begin{array}{ll}
0 & 1 \\
1 & 0
\end{array}\right) .
$$

The conjugacy classes and characters of $\mathrm{D}_{2 N}$ are given in table 2. It can be checked that

Table 2: Character table for $\mathrm{D}_{2 N}$

\begin{tabular}{|c||c|c|c|c|c|}
\hline class & $\{1\}$ & $\begin{array}{c}\left\{\tilde{x}^{k}, \tilde{x}^{-k}\right\} \\
(k \in\{1,2, \ldots, N-1\})\end{array}$ & $\left\{\tilde{x}^{N}\right\}$ & $\begin{array}{c}\left\{y \tilde{x}^{2 l} \mid\right. \\
l=0,1, \ldots, N-1\}\end{array}$ & $\begin{array}{c}\left\{\tilde{y} \tilde{x}^{l+1} \mid\right. \\
l=0,1, \ldots, N-1\}\end{array}$ \\
\hline \hline$\chi_{\circ}$ & 1 & 1 & 1 & 1 & 1 \\
$\chi_{\mathrm{v}}$ & 1 & 1 & 1 & -1 & -1 \\
$\chi_{\mathrm{s}}$ & 1 & $(-1)^{k}$ & $(-1)^{N}$ & -1 & 1 \\
$\chi_{\mathrm{c}}$ & 1 & $(-1)^{k}$ & $(-1)^{N}$ & 1 & -1 \\
$\chi_{m}$ & 2 & $2 \cos (\pi m k / N)$ & $2(-1)^{m}$ & 0 & 0 \\
\hline
\end{tabular}

the representation rings of both $\mathrm{Q}_{N}$ and $\mathrm{D}_{2 N}$ are simply reducible.

Note that for even $N$ the groups $\mathrm{Q}_{N}$ and $\mathrm{D}_{2 N}$ possess identical character tables, and hence in particular identical representation rings. Nevertheless they are not isomorphic; e.g. in $\mathrm{Q}_{N}$ there is only a single element, namely $y^{2}$, of order two, while in $\mathrm{D}_{2 N}$ there are many.

\section{B Characters}

In [9] we considered two species $u^{i}$ and $v^{i}$ of real free fermions. An action of the group $\mathrm{O}(2)$ (which can be interpreted as a DHR gauge group of the free fermion theory) was 
defined by

$$
\gamma_{t}\left(u_{r}^{i}\right)=\cos (t) u_{r}^{i}-\sin (t) v_{r}^{i}, \quad \gamma_{t}\left(v_{r}^{i}\right)=\sin (t) u_{r}^{i}+\cos (t) v_{r}^{i},
$$

where $t \in \mathbb{R}$, and

$$
\eta\left(u_{r}^{i}\right)=u_{r}^{i}, \quad \eta\left(v_{r}^{i}\right)=-v_{r}^{i}
$$

In terms of the complex fermions $b_{r}^{i}$ and $c_{r}^{i}$ (see formula (6.22)) this reads

$$
\gamma_{t}\left(b_{r}^{i}\right)=\mathrm{e}^{\mathrm{i} t} b_{r}^{i}, \quad \gamma_{t}\left(c_{r}^{i}\right)=\mathrm{e}^{-\mathrm{i} t} c_{r}^{i}, \quad \eta\left(b_{r}^{i}\right)=c_{r}^{i}, \quad \eta\left(c_{r}^{i}\right)=b_{r}^{i} .
$$

It is obvious that by restricting this action to the naturally embedded discrete subgroup $\mathrm{D}_{2 N} \subset \mathrm{O}(2)$ we just recover the automorphisms (5.7) and (5.8): $\zeta_{1}=\gamma_{\pi / N}, \vartheta=\eta$.

As in [9] we will use the labelling

$$
\widehat{\mathrm{O}(2)}=\{0, J\} \cup \mathbb{N}
$$

for the group dual of $\mathrm{O}(2)$. In [9] we computed the characters of the sectors of $\mathrm{CAR}^{\mathrm{O}(2)}$; they read

$$
\begin{aligned}
& \chi_{0}^{[\mathrm{O}(2)]}=\frac{\Theta_{0}(q)}{2(\varphi(q))^{2 N}}+\frac{(\varphi(q))^{2 N}}{2\left(\varphi\left(q^{2}\right)\right)^{2 N}}, \quad \chi_{J}^{[\mathrm{O}(2)]}=\frac{\Theta_{0}(q)}{2(\varphi(q))^{2 N}}-\frac{(\varphi(q))^{2 N}}{2\left(\varphi\left(q^{2}\right)\right)^{2 N}}, \\
& \chi_{m}^{[\mathrm{O}(2)]}=\frac{\Theta_{m}(q)}{(\varphi(q))^{2 N}} \quad \text { for } m \in \mathbb{N},
\end{aligned}
$$

with $\varphi$ and $\Theta_{m}$ as defined in (4.15) and (4.16). Now upon induction from $\mathrm{D}_{2 N}$ to $\mathrm{O}(2)$ the irreducible $\mathrm{D}_{2 N}$-representations split into irreducible $\mathrm{O}(2)$-representations as

$$
\begin{aligned}
& \operatorname{ind}_{\mathrm{D}_{2 N}}^{\mathrm{O}(2)}\left(\pi_{\circ}^{\left(\mathrm{D}_{2 N}\right)}\right)=\pi_{0}^{(\mathrm{O}(2))} \oplus \bigoplus_{n=1}^{\infty} \pi_{2 n N}^{(\mathrm{O}(2))}, \quad \operatorname{ind}_{\mathrm{D}_{2 N}}^{\mathrm{O}(2)}\left(\pi_{\mathrm{v}}^{\left(\mathrm{D}_{2 N}\right)}\right)=\pi_{J}^{(\mathrm{O}(2))} \oplus \bigoplus_{n=1}^{\infty} \pi_{2 n N}^{(\mathrm{O}(2))}, \\
& \operatorname{ind}_{\mathrm{D}_{2 N}}^{\mathrm{O}(2)}\left(\pi_{\mathrm{s}}^{\left(\mathrm{D}_{2 N}\right)}\right)=\operatorname{ind}_{\mathrm{D}_{2 N}}^{\mathrm{O}(2)}\left(\pi_{\mathrm{c}}^{\left(\mathrm{D}_{2 N}\right)}\right)=\bigoplus_{n=1}^{\infty} \pi_{(2 n-1) N}^{(\mathrm{O}(2))}, \\
& \operatorname{ind}_{\mathrm{D}_{2 N}}^{\mathrm{O}(2)}\left(\pi_{\alpha}^{\left(\mathrm{D}_{2 N}\right)}\right)=\bigoplus_{n=1}^{\infty}\left(\pi_{2(n-1) N+\alpha}^{(\mathrm{O}(2))} \oplus \pi_{2 n N-\alpha}^{(\mathrm{O}(2))}\right) \quad \text { for } \alpha \in\{1,2, \ldots, N-1\} .
\end{aligned}
$$

As a consequence, we have $\chi_{\circ}^{\left[\mathrm{D}_{2 N}\right]}=\chi_{0}^{[\mathrm{O}(2)]}+\sum_{n=1}^{\infty} \chi_{2 n N}^{[\mathrm{O}(2)]}$ etc., and hence we find the expressions

$$
\begin{aligned}
& \chi_{\circ}^{\left[\mathrm{D}_{2 N}\right]}(q)=\psi_{0}(q) \frac{\Theta_{0}(q)}{2(\varphi(q))^{2 N}}+\frac{(\varphi(q))^{2 N}}{2\left(\varphi\left(q^{2}\right)\right)^{2 N}}, \\
& \chi_{\mathrm{v}}^{\left[\mathrm{D}_{2 N}\right]}(q)=\psi_{0}(q) \frac{\Theta_{0}(q)}{2(\varphi(q))^{2 N}}-\frac{(\varphi(q))^{2 N}}{2\left(\varphi\left(q^{2}\right)\right)^{2 N}}, \\
& \chi_{\mathrm{S}}^{\left[\mathrm{D}_{2 N}\right]}(q)=\chi_{\mathrm{c}}^{\left[\mathrm{D}_{2 N}\right]}(q)=\frac{1}{2} q^{-N / 4} \psi_{N}(q) \frac{\Theta_{N}(q)}{(\varphi(q))^{2 N}}, \\
& \chi_{\alpha}^{\left[\mathrm{D}_{2 N}\right]}(q)=q^{-\alpha^{2} / 4 N} \psi_{\alpha}(q) \frac{\Theta_{\alpha}(q)}{(\varphi(q))^{2 N}}, \quad \alpha \in\{1,2, \ldots, N-1\},
\end{aligned}
$$

with $\psi_{a}$ as defined in (4.17), for the characters of the $\mathrm{CAR}^{\mathrm{D}_{2 N}}$ sectors. By comparison with the characters of the sectors of $\mathrm{Orb}_{N}$ and $\mathrm{SO}_{2 N}$ (given e.g. in [9]), it is then easily checked that indeed the identities (5.17) hold. Moreover, by restricting further the $\mathrm{D}_{2 N}$-action to the $\mathbb{Z}_{2}$-action of $\vartheta$ (corresponding to the element $\tilde{y}$ of $\mathrm{D}_{2 N}$ ), we obtain

$$
\begin{aligned}
& \operatorname{ind}_{\mathbb{Z}_{2}}^{\mathrm{D}_{2 N}}\left(\pi_{+}^{\left(\mathbb{Z}_{2}\right)}\right)=\pi_{\circ}^{\left(\mathrm{D}_{2 N}\right)} \oplus \pi_{\mathrm{c}}^{\left(\mathrm{D}_{2 N}\right)} \oplus \bigoplus_{\alpha=1}^{N-1} \pi_{\alpha}^{\left(\mathrm{D}_{2 N}\right)}, \\
& \operatorname{ind}_{\mathbb{Z}_{2}}^{\mathrm{D}_{2 N}}\left(\pi_{-}^{\left(\mathbb{Z}_{2}\right)}\right)=\pi_{\mathrm{v}}^{\left(\mathrm{D}_{2 N}\right)} \oplus \pi_{\mathrm{s}}^{\left(\mathrm{D}_{2 N}\right)} \oplus \bigoplus_{\alpha=1}^{N-1} \pi_{\alpha}^{\left(\mathrm{D}_{2 N}\right)},
\end{aligned}
$$


where $\pi_{+}^{\left(\mathbb{Z}_{2}\right)} \equiv i d$ and $\pi_{-}^{\left(\mathbb{Z}_{2}\right)}$ denote the two irreducible representations of $\mathbb{Z}_{2}$. The identities (5.13) then immediately follow as a consequence of (5.17).

\section{Acknowledgement.}

It is a pleasure to thank K.-H. Rehren for helpful comments on the manuscript. 


\section{References}

[1] R. Haag, Local Quantum Physics (Springer Verlag, Berlin 1992)

[2] S. Doplicher, R. Haag, and J.E. Roberts, Fields, observables and gauge transformations I. Commun. Math. Phys. 13 (1969) 1

[3] S. Doplicher, R. Haag, and J.E. Roberts, Fields, observables and gauge transformations II. Commun. Math. Phys. 15 (1969) 173

[4] S. Doplicher and J.E. Roberts, Fields, statistics and non-Abelian gauge groups, Commun. Math. Phys. 28 (1972) 331

[5] S. Doplicher and J.E. Roberts, Why there is a field algebra with a compact gauge group describing the superselection structure in particle physics, Commun. Math. Phys. 131 (1990) 51

[6] K.-H. Rehren, Field operators for anyons and plektons, Commun. Math. Phys. 145 (1992) 123

[7] R. Dijkgraaf, C. Vafa, E. Verlinde, and H. Verlinde, The operator algebra of orbifold models, Commun. Math. Phys. 123 (1989) 485

[8] V.G. Knizhnik and A.B. Zamolodchikov, Current algebra and Wess-Zumino model in two dimensions, Nucl. Phys. B 247 (1984) 83

[9] J. Böckenhauer and J. Fuchs, Higher level WZW sectors from free fermions, J. Math. Phys. 38 (1997) 1227

[10] K. Fredenhagen, K.-H. Rehren, and B. Schroer, Superselection sectors with braid group statistics and exchange algebras, II: Covariance properties, Rev. Math. Phys. [special issue] (1992) 111

[11] F. Gabbiani and J. Fröhlich, Operator algebras and conformal field theory, Commun. Math. Phys. 155 (1993) 569

[12] D. Buchholz, G. Mack, and I.T. Todorov, The current algebra on the circle as a germ of local field theories, Nucl. Phys. B (Proc. Suppl.) 5B (1988) 20

[13] K. Hasegawa, Spin module versions of Weyl's reciprocity theorem for classical Kac-Moody Lie algebras - an application to branching rule duality, Publ. RIMS 25 (1989) 741

[14] A.A. Belavin, A.M. Polyakov, and A.B. Zamolodchikov, Infinite conformal symmetry in two-dimensional quantum field theory, Nucl. Phys. B 241 (1984) 333

[15] D. Bernard, V. Pasquier, and D. Serban, Spinons in conformal field theory, Nucl. Phys. B 428 (1994) 612

[16] C.W. Curtis and I. Reiner, Representation Theory of Finite Groups and Associative Algebras (Interscience Publishers, New York 1962) 